\title{
Cardiac and Respiratory Self-Gating in Radial MRI using an Adapted Singular Spectrum Analysis (SSA-FARY)
}

\author{
Sebastian Rosenzweig*1,2, Nick Scholand ${ }^{1,2}$, H. Christian M. \\ Holme $^{1,2}$, and Martin Uecker ${ }^{1,2}$ \\ ${ }^{1}$ Institute for Diagnostic and Interventional Radiology, University \\ Medical Center Göttingen, Göttingen, Germany \\ ${ }^{2}$ German Centre for Cardiovascular Research (DZHK), Partner \\ Site Göttingen, Göttingen, Germany
}

March 31, 2020

\begin{abstract}
Cardiac Magnetic Resonance Imaging (MRI) is time-consuming and error-prone. To ease the patient's burden and to increase the efficiency and robustness of cardiac exams, interest in methods based on continuous steady-state acquisition and self-gating has been growing in recent years. Self-gating methods extract the cardiac and respiratory signals from the measurement data and then retrospectively sort the data into cardiac and respiratory phases. Repeated breathholds and synchronization with the heartbeat using some external device as required in conventional MRI are then not necessary. In this work, we introduce a novel self-gating method for radially acquired data based on a dimensionality reduction technique for time-series analysis (SSA-FARY). Building on Singular Spectrum Analysis, a zero-padded, time-delayed embedding of the auto-calibration data is analyzed using Principle Component Analysis. We demonstrate the basic functionality of SSA-FARY using numerical simulations and apply it to in vivo cardiac radial single-slice bSSFP and Simultaneous Multi-Slice radio-frequency-spoiled gradient-echo measurements, as well as to Stack-of-Stars bSSFP measurements. SSA-FARY reliably detects the cardiac and respiratory motion and separates it from noise. We utilize the generated signals for high-dimensional image reconstruction using parallel imaging and compressed sensing with in-plane wavelet and (spatio-)temporal total-variation regularization.
\end{abstract}

* Sebastian Rosenzweig, University Medical Center Göttingen, Institute for Diagnostic and Interventional Radiology, Robert-Koch-Str. 40, 37075 Göttingen, Germany. sebastian.rosenzweig@med.uni-goettingen.de 
Keywords: Self-Gating, MRI, dimensionality reduction, PCA, Singular Spectrum Analysis

\section{Introduction}

Magnetic Resonance Imaging (MRI) is an intrinsically slow imaging technique, which makes imaging of moving organs particularly challenging. Still, from the early years of MRI, researchers recognized the great chances and implications of monitoring the beating heart without the use of ionizing radiation and with the superior tissue contrast of MRI. Here, the respiratory and cardiac motion pose high demands on the acquisition and reconstruction.

One solution is real-time imaging, which resolves the true dynamics of the heart but is limited in terms of temporal and spatial resolution and restricted to two-dimensional imaging [1, 2, 3, 4]. In clinical practice, pro- or retrospective gating is typically used, which exploits the quasi-periodicity of the respiratory and cardiac motion to compose a single synthetic heartbeat from data acquired during several actual beats. To synchronize data-acquisition with breathing motion, external devices like a respiratory belt or adapted sequences with navigator readouts are commonly used [5, 6]. However, these devices have to be placed and adjusted individually for each patient. Furthermore, the resulting respiratory signal is not always directly correlated to the motion of the heart 77. Sequences with additional interleaved navigator acquisitions prolong the measurement substantially and complicate the use of steady-state sequences. Otherwise, breath-hold commands can be used to avoid the need for respiratory gating completely, which, however, can be exhausting, time-consuming and not expedient for sick or non-compliant patients and children. For cardiac gating, the standard in clinical practice is the use of an electrocardiogram (ECG) [8], but the ECG signals can experience signal distortion when MRI sequences with fast gradient switching are utilized [9].

To avoid these drawbacks and to gain more flexibility, techniques have been developed to extract cardiac motion from the data itself, which is known as retrospective self-gating [10. Similar approaches can also be used to extract respiratory motion [11]. A large number of different strategies for cardiac, respiratory or combined self-gating with Cartesian or Non-Cartesian acquisition were proposed in the past, e.g. [10, 12, 13, 14, 15, 16. Still, the fundamental idea in most approaches is similar: Either a 1D signal is extracted from certain receive channels using a band-pass filter and specific properties of the acquired auto-calibration (AC) data, or a (sliding window) low-spatial high-temporal resolution reconstruction of a specific region of interest (ROI) is analyzed. A more sophisticated yet simple idea was proposed by Pang et al. [17: The general concept of dimensionality reduction [18] is applied to the AC data by using a Principle Component Analysis (PCA) to extract the required motion signals. However, the resulting signals are often spoiled by noise or trajectory-dependent oscillations, which makes additional filtering necessary [19, 20, 21]. Moreover, cardiac and respiratory motion are not always clearly separated [22], which 
complicates data binning into the respective breathing and heart phases and requires the use of further post-processing steps such as coil clustering [23].

To overcome these limitations, we propose the use of an adapted Singular Spectrum Analysis (SSA), which can be thought of as a temporally localized PCA or equivalently as a PCA applied to time-delay embedded coordinates. SSA is an application of the general Karhunen-Loève theorem [24] and a powerful tool for the analysis of dynamical systems, incorporating elements of classical time-series analysis, multivariate statistics, multivariate geometry and signal processing [25. Broomhead and King derived SSA from Takens' theorem for the analysis of chaotic dynamical systems, and applied it to the problems of dynamical systems theory [26, 27. Further development was promoted by Vautard et al. 28, 29]. Since the birth of SSA in 1986 [27, 30] it has found wide-spread application in various fields [31, 32, 33, 34, 35, 36. SSA can be used for noise reduction, detrending and the identification of oscillatory components [29, hence it is ideally suited for the extraction of vital motion signals such as respiratory and cardiac motion in self-gated MRI.

Nevertheless, conventional univariate SSA can only be applied to singlechannel time series, whereas in parallel MRI multiple receive channels (phased array coils) are available. Channels located closer to the heart tend to capture cardiac motion, while coils placed near the diaphragm rather monitor respiratory motion. Manual coil selection [37] can enable the use of univariate SSA for vital motion extraction, but correlated information from other coils is then lost. Moreover, for routine clinical use a fully automated technique is preferred.

Fortunately, univariate SSA has a natural extension for the analysis of a multi-channel time series [30]. However, this multivariate SSA is not a dimensionality reduction technique, but recovers the specific oscillations for each channel rather than to extract a single signal that describes the temporal evolution of the principle motion components.

Here, we adapt the Singular Spectrum Analysis For Advanced Reduction of dimensionalitY, which we dub SSA-FARY [38, 39]. In its original form (multivariate) SSA consists of four steps [25]: I) Hankelization, II) Decomposition, III) Grouping, IV) Backprojection. In SSA-FARY, we remove steps III) and IV) and instead perform a zero-padding operation at the start. We will demonstrate the basic functionality of SSA-FARY in numerical simulations and show reconstructions of in vivo cardiac measurements acquired with single-slice bSSFP and Simultaneous Multi-Slice (SMS) radio-frequency (RF)-spoiled gradient-echo (FLASH) sequences, as well as with a Stack-of-Stars (SOS) bSSFP sequence.

\section{Theory}

In radial single-slice, SMS or Stack-of-Stars imaging the central k-space point or the central line along the slice-dimension $k_{z}\left(k_{x}=0, k_{y}=0\right)$, respectively, have proved to be ideally suited for self-gating [10, 20. We extract this AC data from the measurement data and stack all coils and partitions into a single dimension. This yields a multi-channel time-series $X_{c}^{t}$ of size $\left[\left(N_{p} \times N_{c}\right) \times N_{t}\right]$, 
with $1 \leq t \leq N_{t}$ and $1 \leq c \leq\left(N_{p} \cdot N_{c}\right)$, which contains information about the respiratory and cardiac motion. $N_{t}$ is the total number of central k-space points or lines used for auto-calibration, $N_{p}$ is the number of partitions and $N_{c}$ is the number of receive coils. Each channel $c$ is normalized to have zero mean.

\subsection{Correction of the AC Data}

System imperfections such as gradient delays and off-resonances usually cause a corruption of the AC data $\boldsymbol{X}$, which manifests an oscillation of a trajectorydependent frequency in radial imaging [19. This signal fluctuation is often misinterpreted by dimensionality reduction methods as a major signal contribution. This contribution can mostly be removed by a simple orthogonal projection based on its known frequency. Here, we extend this method to also include higher-order harmonics which yields a method that almost completely removes the unwanted signal. For simplicity, we assume a golden angle acquisition scheme. Let $\varphi_{0}$ be the incremental projection angle, then

$$
\varphi^{t}=t \cdot \varphi_{0}
$$

is the projection angle used for the acquisition at time step $t$. We define the vector

$$
n^{t}:=\left(\begin{array}{c}
e^{i \varphi^{t}} \\
e^{-i \varphi^{t}} \\
e^{i \cdot 2 \varphi^{t}} \\
e^{-i \cdot 2 \varphi^{t}} \\
\vdots \\
e^{i \cdot N_{H} \varphi^{t}} \\
e^{-i \cdot N_{H} \varphi^{t}}
\end{array}\right)
$$

containing the oscillations up to the $N_{H}$-th harmonic as a basis for the perturbing oscillation and constrain $\boldsymbol{X}$ to be orthogonal to $\boldsymbol{n}$,

$$
\boldsymbol{X}_{\text {cor }}=\boldsymbol{X}_{\text {raw }}-\boldsymbol{n}\left(\boldsymbol{n}^{\dagger} \boldsymbol{X}_{\text {raw }}\right),
$$

with $^{\dagger}$ denoting the pseudo-inverse. This procedure cleans the corrupted signal $\boldsymbol{X}_{\text {raw }}$ and yields a corrected time series $\boldsymbol{X}_{\text {cor }}$. We use this AC correction method in all presented in vivo experiments.

\subsection{Dimensionality Reduction Methods}

Principle Component Analysis PCA can be understood as the rotation of the original coordinate system to a new one with orthogonal axes that coincide with the directions of maximum variable variance [40. The PCA of a time series $\boldsymbol{X}$ can be performed using the Singular Value Decomposition (SVD).

$$
\boldsymbol{X}^{T}=\boldsymbol{U} \boldsymbol{S} \boldsymbol{V}^{H} .
$$


Here, the diagonal matrix $\boldsymbol{S}$ contains the real eigenvalues $\lambda_{1} \geq \cdots \geq \lambda_{N_{t}} \geq 0$ in decreasing order of magnitude. PCA provides the expansion of $\boldsymbol{X}^{T}$ onto the orthonormal $\left[N_{t} \times N_{t}\right]$ basis $\boldsymbol{U}$,

$$
\left(X^{T}\right)_{t}=\sum_{k=1}^{N_{t}} U_{t}^{k}\left(S V^{H}\right)_{k},
$$

where the principle components $\left(S V^{H}\right)_{k}$ are given by

$$
\left(S V^{H}\right)_{k}:=\sum_{l=1}^{N_{c} \cdot N_{p}} S_{k}^{l}\left(V^{H}\right)_{l}=\lambda_{k}\left(V^{H}\right)_{k} .
$$

Since the cardiac and respiratory motion signals contribute as main sources of variation to the time series $\boldsymbol{X}$, their temporal behavior should be captured by one of the first basis vectors $U^{k}$, respectively [17].

Singular Spectrum Analysis For Advanced Dimensionality Reduction (SSA-FARY) A schematic of the SSA-FARY procedure is depicted in Fig. 1a. In contrast to conventional (multivariate) SSA, we first zero-pad $(\mathcal{Z})$ the second dimension of the AC data $\boldsymbol{X}$ to obtain matrix $\tilde{\boldsymbol{X}}$ of size $\left[\left(N_{p} \times N_{c}\right) \times\left(N_{t}+W-1\right)\right]$,

$$
\tilde{\boldsymbol{X}}=\mathcal{Z} \boldsymbol{X}
$$

Next, we construct a Block-Hankel calibration matrix

$$
\boldsymbol{A}=\mathcal{H} \tilde{\boldsymbol{X}}
$$

of size $\left[N_{t} \times\left(\left(N_{p} \times N_{c}\right) \times W\right)\right]$. Here, the Hankelization operator $\mathcal{H}$ slides a window of size $[1 \times W]$ through channel $\tilde{X}_{c}$ of the zero-padded AC data and takes each block to be a row in the $c$-th column of the calibration matrix. This operation is similar to the construction of the calibration matrix in ESPIRiT [41.

We decompose $\boldsymbol{A}$ using an SVD

$$
\boldsymbol{A}=\boldsymbol{U} \boldsymbol{S} \boldsymbol{V}^{H}
$$

and consider $\boldsymbol{U}$ of size $\left[N_{t} \times N_{t}\right]$ as the orthonormal basis that consists of the Empirical Orthonormal Functions (EOFs) $U^{k}, 1 \leq k \leq N_{t}$. The principle components $\left(S V^{H}\right)_{k}$ are given by

$$
\left(S V^{H}\right)_{k}:=\sum_{l=1}^{W \cdot N_{c} \cdot N_{p}} S_{k}^{l}\left(V^{H}\right)_{l}=\lambda_{k}\left(V^{H}\right)_{k} .
$$

The expansion of $\boldsymbol{A}$, or $\tilde{\boldsymbol{X}}$, in the basis $\boldsymbol{U}$ then reads

$$
\tilde{X}_{c}^{t+j}=A_{t}^{c j}=\sum_{k=1}^{N_{t}} U_{t}^{k}\left(S V^{H}\right)_{k}^{c j},
$$


where $1 \leq t \leq N_{t}$ iterates through the temporal samples, $1 \leq c \leq\left(N_{c} \cdot N_{p}\right)$ through the channels and $0 \leq j<W$ is the index inside the sliding window. The EOFs can be considered as data-adaptive weighted moving averages of the original time series $\tilde{\boldsymbol{X}}$, with $\boldsymbol{V}$ being the data-adaptive filters [29, 42],

$$
\begin{gathered}
\boldsymbol{U} \boldsymbol{S}=\boldsymbol{A} \boldsymbol{V}, \\
U_{t}^{k}=\frac{1}{\lambda_{k}} \sum_{c=1}^{N_{p} \cdot N_{c}} \sum_{j=1}^{W} \tilde{X}_{c}^{t+j} V_{c j}^{k} .
\end{gathered}
$$

In fact, the columns of $\boldsymbol{V}$ can bee seen as a complete eigenfilter decomposition of the original time series [43. These filters $V^{k}$ act as data-adaptive band-pass filters with a frequency bandwidth $\delta f_{\mathrm{B}}$ given by

$$
\delta f_{\mathrm{B}}=\frac{f_{\mathrm{s}}}{W},
$$

where $f_{\mathrm{s}}$ is the sampling rate [44, 45]. Harris and Yuan showed in 42] for the univariate case that a periodic oscillation contained in the data lead to an even and odd filter. The application of these filters to the original time series constitutes for each oscillation one EOF which is in phase and one which is in quadrature to the original oscillation, respectively.

Vautard et al. 29] proposed another interpretation of the EOFs considering the minimization problem

$$
\left.\underset{\alpha}{\arg \min }\right|_{t, k} \sum_{c=1}^{N_{p} \cdot N_{c}} \sum_{j=1}^{W}\left\|\tilde{X}_{c}^{t+j}-\alpha\left(S V^{H}\right)_{k}^{c j}\right\|^{2} .
$$

The solution of eq. 15 is $\alpha=U_{t}^{k}$, thus the EOFs can be obtained by a local least-squares fit of the $\mathrm{k}$-th principle component to the original time series. This locality, determined by the window size $W$, distinguishes SSA-FARY from classical PCA, which does not take the temporal past and future of a sample into account. In fact, PCA is a special case of SSA-FARY with $W=1$,

$$
\left.\boldsymbol{A}\right|_{W=1}=\mathcal{H} \tilde{\boldsymbol{X}}=\mathcal{H} \mathcal{Z} \boldsymbol{X}=\boldsymbol{X}^{T} .
$$

In conventional SSA, i.e. where no zero-padding is applied, the EOFs are of reduced length $N_{t}-W+1$. Thus, the exact correspondence in time is lost, which inhibits their use as self-gating signal. In contrast, the EOFs $U^{k}$ in SSAFARY preserve the length $N_{t}$ of the original time series and can directly be used for self-gating, similar to the eigenvectors $U^{k}$ in PCA. In distinction to PCA, the EOFs in SSA(-FARY) capture temporal oscillations via oscillatory pairs 28. In particular, if two consecutive eigenvalues are nearly equal, the two corresponding EOFs are nearly periodic with the same period and in quadrature [46], which is a consequence of the filtering property of SSA-FARY 42]. To ensure a proper separation the singular values of different EOF pairs should 
be distinct, which is called the strong separability condition [47] and usually fulfilled for our application.

These pairs can be seen as the data-adaptive equivalent to the sine-cosine pairs of Fourier analysis [48. A single EOF-pair might suffice for the analysis of nonlinear and inharmonic oscillations, as it automatically locates intermittent oscillatory regions. In contrast, classical spectral analysis would require a large amount of harmonics or subharmonics of the fundamental period [29, 49].

Comments The EOF $U^{k}$ is also the k-th left eigenvector of the $\left[N_{t} \times N_{t}\right]$ real-symmetric cross-covariance matrix

$$
C=A A^{H} .
$$

Depending on the number of acquired spokes and partitions, computing the eigen-decomposition of $\boldsymbol{C}$ is usually more efficient than computing the SVD of A.

In contrast to the parameter-free PCA, for SSA we must define a window size $W$. To long-range correlations in time, $W$ should be large, which - as a trade-off - results in a lower degree of statistical confidence [49]. Vautard et al. [29] showed that SSA can resolve oscillations best when the periods are shorter than the window size $W$. In our study, the window size $W \approx 3$ s proofed to be a robust choice for most measurements, independently of the utilized sequence, and this value was chosen as the default. More information on the choice of the window size is given in the Methods and Discussion section.

The fundamental concept behind the use of a temporal window $W$ is Taken's delay embedding theorem [26, one of the backbones of chaotic dynamical system analysis. Instead of considering each temporal sample individually and isolated from other time points, so called time-delay coordinates are constructed by embedding the samples in a higher-dimensional space with embedding dimension $W$. Consequently, each time point is represented by a time-delay coordinate vector, which comprises not only the sample of the respective time but also its temporal past and future. It is therefore a natural choice to pick an odd value for $W$ in order to incorporate the same amount of past and future information.

Towards the beginning and the end of a time series this embedding can no longer be constructed due to lack of past or future samples, respectively. There are two strategies to overcome this limitation: 1.) Time-delay coordinates are constructed for the central $N_{t}-(W-1)$ samples only, which means that $W-1$ samples would be discarded from future processing. 2.) $(W-1) / 2$ samples are zero-padded on both ends of the time series, which comes at the expense of increased inaccuracy for the marginal samples of the time series. However, for the second approach no samples have to be discarded and through the symmetric zero-padding the time-delay coordinates remain in sync with the actual temporal evolution of the signal. In this manuscript, the second approach is used. 


\subsection{Binning}

The myocardium shows different behavior for contraction and expansion, so usually the entire cardiac cycle is divided into multiple distinct bins to accurately resolve the temporal motion. For respiratory gating it is usually assumed that inspiration and expiration do not have to be distinguished [50, 20, 16. However, various studies reveal that respiratory motion is heavily subject-dependent and exhibits a strong variability as well as hysteresis, which affects the global position of the myocardium [51, 52, 53, 54]. Hence, inspiration and expiration should be distinguished to properly resolve the effects of breathing motion on the heart.

Since SSA-FARY yields EOF quadrature pairs that capture the phase information of periodic oscillations, binning is straight-forward for both cardiac and respiratory motion: The phase portrait, i.e. the amplitude-amplitude scatter plot, of an EOF pair is divided into $N$ circular sectors with central angle $\phi=360^{\circ} / N$. The samples are then binned according to their respective circular sector, see Fig. 1 $1 \mathrm{~b}$.

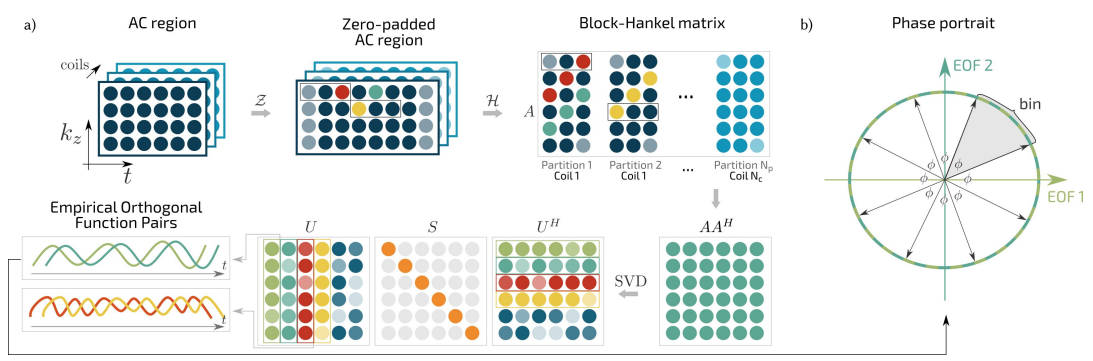

Figure 1: (a) Schematic of SSA-FARY: The multi-channel AC data is zeropadded $\mathcal{Z}$ and Hankelized $\mathcal{H}$. Then the cross-correlation matrix $A A^{H}$ is calculated and decomposed using an eigenvalue or singular value decomposition. The left eigenvector $U$ contains the EOF quadrature pairs that represent the principle motion signals of the time series. (b) Binning: The phase portrait of an EOF quadrature pair is divided into $N$ circular sectors with equal central angle $\varphi=360^{\circ} / N$. The samples are then assigned to bins according to their respective circular sector.

\section{Methods}

\subsection{Numerical Simulations}

We compare the capability of PCA and SSA-FARY in extracting and separating oscillatory signals in simple numerical simulations.

The signals we want to extract are two frequency-modulated sinusoids

$$
\begin{gathered}
a(t)=A \sin \left(\varphi_{a}+\omega_{a} t+\phi_{a}(t)\right), \\
b(t)=B \sin \left(\varphi_{b}+\omega_{b} t+\phi_{b}(t)\right),
\end{gathered}
$$


with

$$
\begin{gathered}
\phi_{a}(t)=\Phi \sin (2 \pi t / 2 T), \\
\phi_{b}(t)=\Phi \sin (2 \pi t / T),
\end{gathered}
$$

which account for frequency variations. To simulate various channels $i$, we use a weighted sum

$$
x_{i}(t)=\frac{i}{N_{c}} a(t)+\frac{\left(N_{c}+1-i\right)}{N_{c}} b(t), \quad 1 \leq i \leq N_{c} .
$$

To spoil the composite signals $x_{i}(t)$, we add Gaussian white noise $h_{\text {noise }}(t)$ with standard deviation $\sigma_{\text {noise }}$, or an oscillatory spell from time $t_{1}$ to time $t_{2}$,

$$
h_{\text {spell }}(t)=C \sin \left(\varphi_{c}+\omega_{c} t\right), \quad t_{1} \leq t \leq t_{2},
$$

or an exponential trend

$$
h_{\text {trend }}(t)=D_{1} e^{\xi t}-D_{2},
$$

to all channels, which yields

$$
X_{i}(t)=x_{i}(t)+h(t) .
$$

We analyze the time-series $\boldsymbol{X}^{\text {noise }}, \boldsymbol{X}^{\text {spell }}$ and $\boldsymbol{X}^{\text {trend }}$ using PCA and SSAFARY with window size $W$. Note, that the aim of this numerical experiment is to demonstrate the general benefits of SSA-FARY over PCA for the analysis of time series, and not to simulate cardiac and respiratory motion in the most accurate way. We therefore did not include the modeling of a more complex frequency variability or motion signal shapes. More details on the simulation are provided in the appendix.

\section{$3.2 \quad$ In Vivo experiments}

All measurements were performed on a Skyra 3T scanner (Siemens Healthcare GmbH, Erlangen, Germany) using 30 channels of a thorax and spine coil. Gradient delay correction was performed using RING [55, 56. The AC data was corrected using the orthogonal projection with $N_{H}=5$. In the following, the $\mathrm{AC}$ data's real and imaginary part are treated as individual channels. The field of view in all experiments was $256 \times 256 \mathrm{~mm}^{2}$ at base resolution 192. All presented experiments were performed on volunteers with no known diseases, who gave written informed consent. All SOS measurements were performed on different volunteers. The study had received approval from the local ethics committee.

Sequence Design, Auto-Calibration and Reconstruction We utilize a radial bSSFP sequence for the single-slice measurement, an RF-spoiled gradientecho sequence with randomized RF spoiling [57] for the SMS measurement and a radial bSSFP sequence with undersampling in $k_{z}$ direction 58 for the SOS measurement. To obtain maximum $\mathrm{k}$-space coverage and thus improved image 
quality [59, 60], the projection angle $\varphi$ is increased in each shot about the seventh tiny golden angle $\varphi_{0} \approx 23.6^{\circ}[61$. For the SMS and SOS measurements, the partitions are acquired in an interleaved fashion, i.e. one spoke is recorded for each partition before the next in-plane spoke of a partition is acquired.

For single-slice imaging, the central sample of all spokes is used for autocalibration. For SMS, more AC data is available as not only a single sample but the central line along the $k_{z}\left(k_{x}=0, k_{y}=0\right)$ direction can be utilized.

Inspired by [58, we make use of variable-density $k_{z}$-undersampling for the SOS acquisition. From the total number of 14 partitions, the central 6 partitions are always acquired and the corresponding central line is used for autocalibration. The remaining 8 partitions are undersampled by a factor of 4 . This center-dense sampling scheme does not only increase the temporal resolution of the $\mathrm{AC}$ data but also improves the image quality 62 .

For self-gating with PCA and SSA-FARY as well as for imaging reconstruction we use BART 63. Image reconstruction is performed using combined parallel imaging and compressed sensing (PICS) 64 applying the alternating direction method of multipliers (ADMM) 65] with in-plane wavelet-regularization on the spatial dimensions and total variation (TV) on the cardiac and respiratory dimension [20, 66]. For SOS imaging, we additionally apply TV regularization in slice direction. The coil sensitivities for the single-slice and SMS measurements are generated using radial ENLIVE allowing two maps [3, 60, 67, 41]. To reduce the memory demand we allow only one map in the SOS reconstruction. We apply coil compression [68, 69 to reduce the number of coils to 13 for single-slice and SMS, and 10 for SOS imaging and perform the calibration of the sensitivities using a lower resolution.

The SSA-FARY gating signals is distributed into 25 cardiac and 9 respiratory bins for image reconstruction. Although not always necessary, we standardly perform an additional detrending of the EOFs using a moving average filter of length $L_{\text {avg }} \approx 3 W$. This further improves the binning accuracy of SSA-FARY by removing a possibly remaining residual trend.

In the spirit of reproducible research, code and data to reproduce the experiments are made available on Github 1

Single-slice imaging We perform a 72 second free-breathing bSSFP scan $\left(\mathrm{TE} / \mathrm{TR}=1.90 / 3.80 \mathrm{~ms}\right.$, flip angle $\left.40^{\circ}\right)$ with slice-thickness $7 \mathrm{~mm}$ of the human heart in short-axis view and use the first 30 seconds of data for further analysis and image reconstruction. The full 72 second scan is used for a gridding reconstruction in Supplementary Material chapter II. We furthermore conduct an ECG-triggered CINE bSSFP breath-hold scan of the same slice $(\mathrm{TE} / \mathrm{TR}=$ $1.52 / 3.04 \mathrm{~ms}$, flip angle $57^{\circ}$, slice-thickness $7 \mathrm{~mm}$ ).

We compare the principal motion signals using PCA and SSA-FARY with window size $W=751$, which covers a period of about $3 \mathrm{~s}$.

1 https://github.com/mrirecon/SSA-FARY 
SMS imaging We perform a 60 second free-breathing SMS RF-spoiled gradientecho scan $\left(\mathrm{TE} / \mathrm{TR}=1.79 / 2.90 \mathrm{~ms}\right.$, flip angle $\left.12^{\circ}\right)$ with three simultaneously acquired slices in short-axis view. The slice thickness is $5 \mathrm{~mm}$ and the slice gap $10 \mathrm{~mm}$. We use SSA-FARY with window size $W=345$, which covers a period of about $3 \mathrm{~s}$. We perform a joint reconstruction of all slices using binning based on SSA-FARY. To evaluate the accuracy of SSA-FARY, we compare the SSA-FARY respiration quadrature-signals of the complete time series with the breathing pattern extracted from a pneumatic respiratory belt (Siemens Healthcare GmbH, Erlangen, Germany) and a real-time reconstruction [60, 70].

SOS imaging We measure 8 volunteers and on each we perform a freebreathing three-minute radial SOS bSSFP scan $(\mathrm{TE} / \mathrm{TR}=1.90 / 3.80 \mathrm{~ms}$, flip angle $35^{\circ}$ ) with fourteen partitions in short-axis view and slice thickness $7 \mathrm{~mm}$. By default we use SSA-FARY with window size $W=91$, which covers a period of about $2.8 \mathrm{~s}$. For one volunteer the respiratory EOF pair revealed highly irregular breathing with occasional breath-holds of up to $12 \mathrm{~s}$ and for another volunteer the cardiac EOF pair showed a pronounced frequency variation. To improve the gating accuracy in these two cases, we determined another respiratory EOF pair using window size $W=51$ and another cardiac EOF pair using window size $W=21$, respectively.

To evaluate the precision of the cardiac gating with SSA-FARY, we analyze the SSA-FARY signal in comparison to the simultaneously acquired ECG trigger using a Python 3 script. Therefore, we define a synthetic trigger point when the phase related to the orthogonal cardiac SSA-FARY quadrature pair experiences a zero-phase crossing. Since the global phase-offset of the quadrature pair is arbitrary, we correct the synthetic SSA-FARY trigger by a constant shift using the average distance to the ECG trigger. We then compute the standard deviation $\sigma_{\text {trig }}$ and standard error of the corrected SSA-FARY trigger to the ECG trigger.

Moreover, we acquire the same slices using a conventional ECG-triggered breath-hold CINE single-slice stack bSSFP measurement with Cartesian readout $(\mathrm{TE} / \mathrm{TR}=1.52 / 3.04 \mathrm{~ms}$, flip angles depending on the Specific Absorbtion Rate (SAR) limits between $47^{\circ}$ and $62^{\circ}$ ) and cardiac bin size $T_{\text {bin }} \approx 48 \mathrm{~ms}$. The patient dependent measurement time is around $6-8 \mathrm{~min}$. We compare the enddiastolic and end-systolic left-ventricular blood-pool area of a mid-ventricular slice to the SSA-FARY based reconstruction using Image J.

\section{Results}

Numerical simulations Fig. 2 depicts the results of the numerical simulations. PCA is able to extract, at least essentially, the shape of the two oscillations $a(t)$ and $b(t)$ from $\boldsymbol{X}^{\text {noise }}$ and $\boldsymbol{X}^{\text {spell }}$. However, both the noise and the oscillation spell are still evident in the resulting eigenvectors and corrupt the signal. PCA fails to produce a useful result for $\boldsymbol{X}^{\text {trend }}$. While oscillation $a(t)$ is 
present in two principle eigenvectors, oscillation $b(t)$ is not distinctly separated in any of the eigenvectors.

In contrast, SSA-FARY extracts the oscillation signals with almost no spoiling residuals in all three investigated cases. Only at the borders deviations from the ideal signal can be observed. Notably, SSA-FARY does not only yield a one-dimensional signal of the temporal evolution of an oscillation, but preserves the phase information by quadrature pairs, as can be appreciated from the amplitude-amplitude plots. The two EOFs corresponding to the same pair have very similar singular values. In the scree plot of Fig. 2 b) and c), the first plateau corresponds to EOF 1 and 2, and the second plateau belongs to EOF 3 and 4. In Fig. 2 d), the first two plateaus correspond to EOF 1 and 2, and to EOF 4 and 5. Hence, SSA-FARY does not mix the trend into the oscillations but creates an additional EOF to account for it 2

\footnotetext{
${ }^{2}$ In Supplementary Material Fig. 1-3 we provide results for simulations with different noise variance and trend and spell amplitudes, as well as a different frequency variation. More information on these figures is provided in chapter I of the supplementary document.
} 

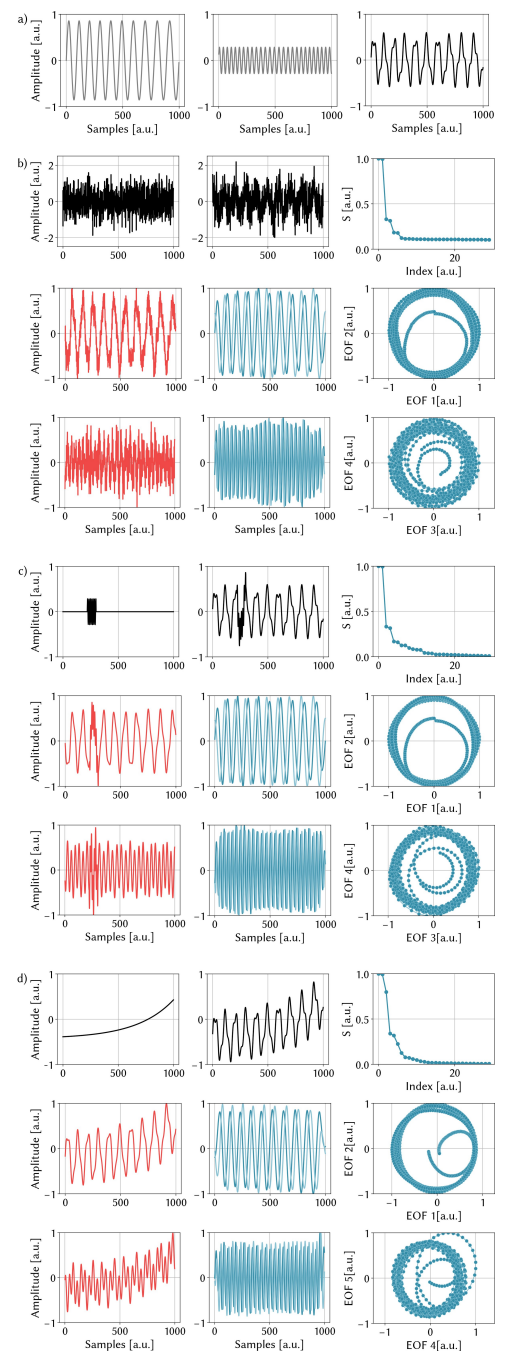

Figure 2: Comparison of PCA eigenvectors and SSA-FARY EOFs. a) Frequency-modulated sinusoidal oscillations $(a(t)$ left, $b(t)$ center) used for the numerical simulations and weighted composite signal (right). b), c), d) Black: Different additional signals (left) - b) noise, c) oscillatory spell, d) exponential trend - added to the composite signal of a) and a selected channel of the resulting signal $X_{i}(t)$ (right). Red: First two eigenvectors of the PCA. Blue: SSA-FARY singular values $S$ (scree plot) and EOF pairs (amplitude-time plot and amplitude-amplitude scatter plot). All plots are normalized. 
Single-slice imaging For each coil, Fig. 3 depicts the DC component of 100 consecutive spokes before and after the data correction using the orthogonal projection. Before correction some coils exhibit pronounced oscillations with approximately 15 samples per period. As we have used the seventh tiny golden angle $\left(\varphi_{0} \approx 23.6^{\circ}\right)$ the oscillations period of 15 samples corresponds to $\varphi^{15}=$ $15 \cdot \varphi_{0} \approx 354^{\circ}$ (see eq. (1)). Hence, the oscillation period in the AC data is linked to the period of the projection angle. By removing this frequency and the higher-order harmonics these oscillations can be completely eliminated.

Fig. 4 shows the self-gating signals generated with PCA and SSA-FARY. In SSA-FARY, the first two EOFs represent respiratory motion and the third and fourth EOF cardiac motion. The EOFs of the pairs are in quadrature, respectively. Both the cardiac and the respiratory phases are well separated. In contrast, PCA cannot fully extract and separate the signals as respiratory and cardiac motion are superposed and heavily spoiled by noise.

Fig. 44 shows six representative images of the SSA-FARY-gated reconstruction. Depicted are from bottom to top the end-systolic, an intermittent and the end-diastolic frame for end-expiration and end-inspiration, respectively. For comparison, we also show the result of the ECG-triggered CINE breath-hold scan.

In Supplementary Material chapter II we present the results of a conventional gridding reconstruction of the full 72 second measurement gated with SSAFARY 3

In Supplementary Material chapter III we present a similar experiment with a RF-spoiled gradient-echo sequence 4

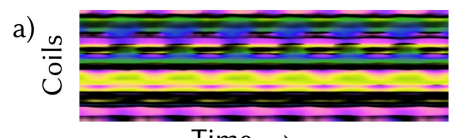

Time $\rightarrow$

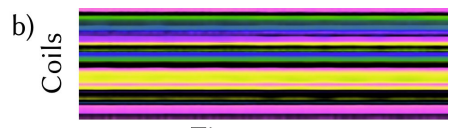

Time $\rightarrow$

Figure 3: Snippet of the amplitude plot with color-coded phase of the DC samples used for auto-calibration of the single-slice reconstruction before (a) and after (b) the data correction using the orthogonal projection. The period length of the oscillations in (a) corresponds to 15 samples.

\footnotetext{
${ }^{3}$ Supplementary Material Fig. 4 shows the SSA-FARY and PCA gating signals of the 72 second scan. Moreover, a gridding reconstruction for three different cardiac phases is depicted and the results of a breath-hold and an ECG-gated CINE scan are shown for comparison.

${ }^{4}$ Supplementary Material Fig. 5 shows the effect of the proposed correction on the AC region. Supplementary Material Fig. 6 shows the self-gating signals determined with SSA-FARY and PCA. Furthermore, six representative frames of the SSA-FARY-gated reconstruction for different respiratory and cardiac motion states are depicted.
} 

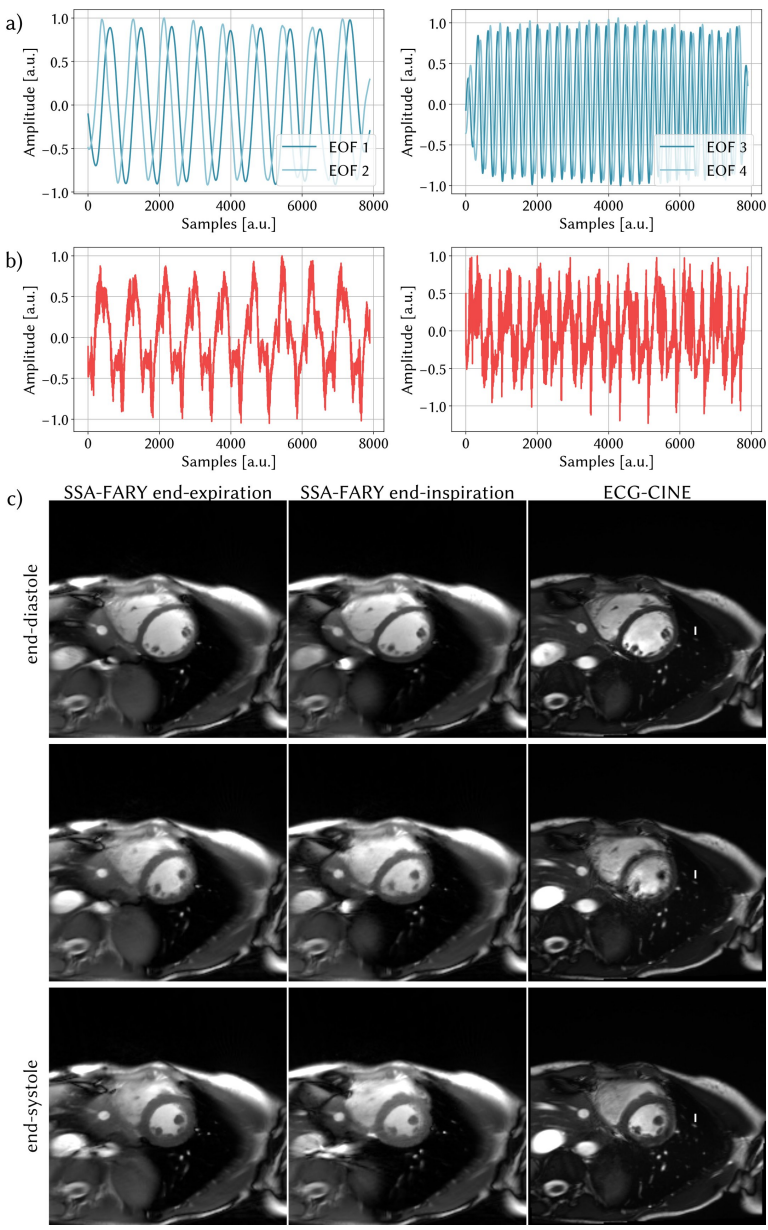

Figure 4: Self-gating signals and PICS reconstruction of the human heart from a single-slice radial bSSFP acquisition in comparison to a conventional CINE scan. In (a) the two quadrature EOFs of SSA-FARY are plotted against time for the respiratory (left) and cardiac (right) motion, respectively. In (b) the first two eigenfunctions of the PCA are depicted. In both, a superposition of the cardiac and respiratory motion spoiled by additional noise can be perceived. For (c) the self-gating signal of (a) was utilized. It shows 6 representative frames of the PICS reconstruction using SSA-FARY corresponding to three cardiac phases from end-systole to end-diastole at end-expiration and end-inspiration. For comparison, we also show the vendor images of an ECG-triggered CINE breath-hold scan. 
SMS imaging Fig. 5 a shows 5 out of 25 cardiac phases for all three slices in end-expiration. The different systolic and diastolic phases are well resolved.

The background of Fig. 5p shows the temporal evolution of a line extracted from an SMS-NLINV real-time reconstruction of the full time series. The line was placed in slice two in vertical direction such that the actual motion of the diaphragm can be observed. This diaphragmatic motion is linearly related to the translation of the heart during breathing [71]. On top of the background image, we have plotted the respiratory EOF quadrature pair obtained from SSAFARY as well as the signal provided by the respiratory belt. For the purpose of comparison, the motion signals were scaled according to the amplitude of the diaphragm motion in the background image.

One of the EOFs (light blue) coincides very well both in amplitude and phase with the temporal evolution of the diaphragm. This is in line with the filtering interpretation of SSA-FARY, which states that one EOF of the quadrature pair is in phase with the related underlying motion, whereas the other EOF (dark blue) constitutes the quadrature signal. The in-phase EOF is furthermore in good agreement with the motion signal provided by the respiratory belt.

The presented volunteer shows a largely periodic breathing pattern. In Supplementary Material chapter IV we present the results of the same experiment on another volunteer, which happend to exhibit a highly irregular respiration pattern during measurement:5

${ }^{5}$ In Supplementary Material Fig. 7 we provide results of the same experiment conducted on a different volunteer. We depict 5 cardiac phases for all three slices, as well as a comparison between the SSA-FARY respiratory self-gating signal, the respiratory belt and the diaphragm motion extracted from a real-time reconstruction. 


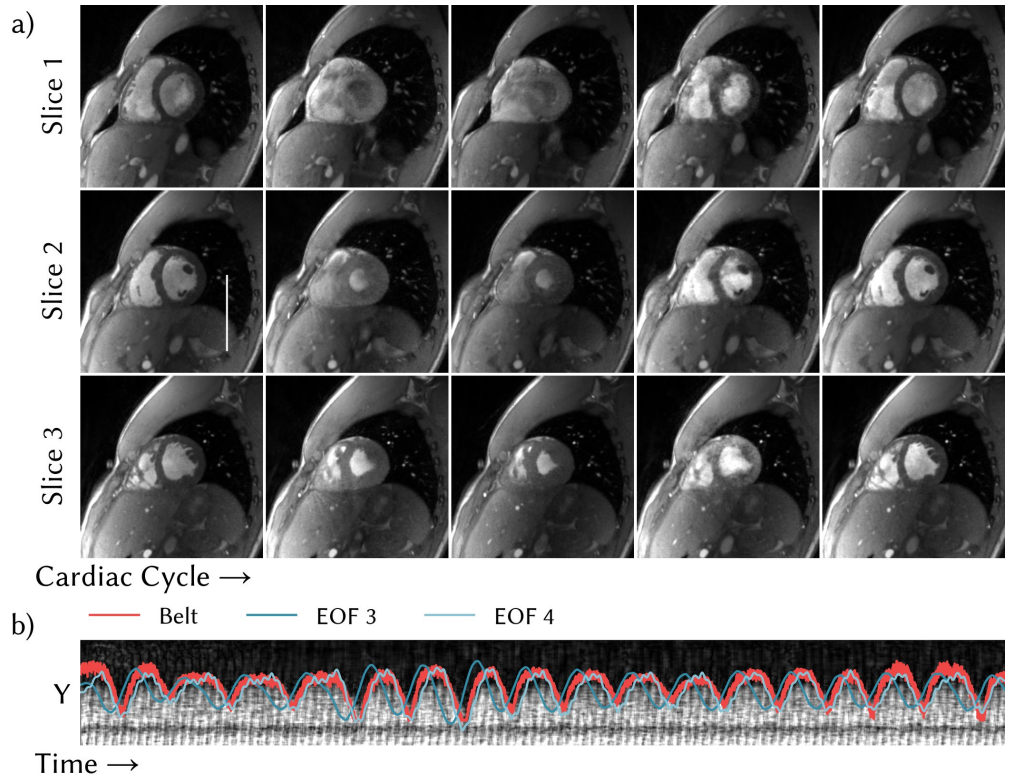

Figure 5: Images of the human heart reconstructed using PICS and the SSAFARY self-gating signal from a radial SMS gradient-echo acquisition. (a) shows 5 out of 25 cardiac phases in end-expiration for each of the three slices. The background of (b) is extracted from an SMS-NLINV real-time reconstruction of the entire time series and shows the temporal evolution of a vertical line (highlighted in white), placed on the diaphragm in slice two. On top, the respiratory self-gating EOF quadrature pair of SSA-FARY and the signal extracted from the respiratory belt is plotted. The amplitudes of the motion signals are scaled for the purpose of comparison.

SOS imaging Fig. 6a depicts a zoomed view on EOFs representing respiratory and cardiac motion for different window sizes. For window size $W=31$, which - considering the undersampling scheme - covers a period of $\approx 1.0 \mathrm{~s}$, respiratory and cardiac motion are not fully separated and appear superposed in one EOF for respiratory and one EOF for cardiac motion. In contrast, for the proposed window size $W=91(\approx 2.8 \mathrm{~s})$ the motion signals are well separated. Then again, for $W=151(\approx 4.5 \mathrm{~s})$ a signal loss can be observed in the cardiac EOFs. Fig. 6b shows one respiratory EOF and one cardiac EOF for the windows $W=81, W=91$ and $W=101$, covering periods from $2.5 \mathrm{~s}$ to $3.0 \mathrm{~s}$. All signals are in good agreement. The corresponding pairing-components of the EOFs show similar behavior and are therefore not depicted. Fig. 6. presents end-diastolic and end-systolic frames for three out of fourteen slices in endexpiration. All respiratory and cardiac states are well separated, the cardiac wall and the diaphragm are sharply resolved. Note, however, that some slices at the fringe of the slab have low signal intensity due to an unoptimized ex- 
citation profile. The image quality is comparable to the CINE reconstruction Fig. 6 $\mathrm{d}$, although the latter tends to be sharper. Due to the higher flip-angle, which is restricted by SAR limitations in volumetric sequences, the CINE images possess a better blood myocardium contrast.

For the different volunteers, Table (1) shows the average heart-rate $\bar{f}_{\text {heart }}$, its standard deviation $\sigma_{\text {heart }}$, the standard deviation $\sigma_{\text {trig }}$ of the synthetic SSAFARY trigger to the ECG trigger and the end-diastolic and end-systolic leftventricular blood-pool area of a mid-ventricular slice for CINE and SSA-FARY.

For imaging on a $3 \mathrm{~T}$ system an insufficient shim can lead to banding artifacts. Measurements with bandings affecting the heart could not immediately be noticed and repeated as the reconstruction was performed offline. Therefore, these measurements were discarded for which the analysis of one volunteer is omitted.

The observed average heart rates range from $0.91 \mathrm{~Hz}(54.60 \mathrm{bpm})$ to $1.38 \mathrm{~Hz}$ $(82.20 \mathrm{bpm})$ with different heart rate variabilities. For the given SOS acquisition with 14 partitions (6 AC lines and undersampling factor of 4), the temporal resolution of the SSA-FARY trigger is $30.4 \mathrm{~ms}$. The standard deviation of the SSA-FARY trigger to the ECG trigger, $\sigma_{\text {trig }}$, is of similar size. Hence, the SSAFARY trigger is in good agreement with the ECG signal and also matches the temporal resolution of the ECG-CINE acquisition.

The areas of the chosen mid-ventricular slices are comparable for ECG-CINE and SSA-FARY, particularly for end-diastole the difference lies mostly in the lower single-digit percent range, whereas a larger uncertainty can be observed for end-systole.

Volunteer V6 exhibits a highly erratic breathing pattern and volunteer V7 possesses a strongly irregular heartbeat and furthermore unintentionally yawned three times during the measurement. Still, SSA-FARY can provide satisfying results as Table 1 and the figures in the Supplementary Material show 6

For all in vivo experiments, we have attached representative movies as Supplementary Material. 7

\footnotetext{
${ }^{6}$ Supplementary Material Fig. 8 and 9 display the SSA-FARY gating signal, representative frames of the SSA-FARY-based image reconstruction and the corresponding CINE reconstructions for volunteers V6 and V7. More information on the figures is provided in chapter V of the supplementary document.

${ }^{7}$ The files Mov1-14 of the Supplementary Material show representative movies of all in vivo reconstructions. More information on the movies is provided in chapter VI of the supplementary document.
} 
Table 1: Average heart rate $\bar{f}_{\text {heart }}$ and corresponding standard deviation $\sigma_{\text {heart }}$, standard deviation of SSA-FARY from ECG trigger $\sigma_{\text {trig. }}$. End-systolic and end-diastolic left-ventricular blood-pool area of a mid-ventricular slice for ECGCINE and SSA-FARY and corresponding error. ${ }^{*}$ denotes the volunteer with highly erratic respiration, ${ }^{* *}$ denotes the volunteer who yawned.

\begin{tabular}{|c|c|c|c|c|c|c|c|c|c|}
\hline \multirow[t]{2}{*}{ Volunteer } & \multirow[t]{2}{*}{$\bar{f}_{\text {heart }}[\mathrm{Hz}]$} & \multirow[t]{2}{*}{$\sigma_{\text {heart }}[\mathrm{Hz}]$} & \multirow[t]{2}{*}{$\sigma_{\text {trig }}[\mathrm{ms}]$} & \multicolumn{3}{|c|}{ Diastole $\left[\mathrm{mm}^{2}\right]$} & \multicolumn{3}{|c|}{ Systole $\left[\mathrm{mm}^{2}\right]$} \\
\hline & & & & ECG & SSA-FARY & Err. [\%] & ECG & SSA-FARY & Err. [\%] \\
\hline V1 & $0.910(4)$ & $0.057(3)$ & $23(1)$ & 2254 & 2222 & 1.4 & 1068 & 1017 & 4.8 \\
\hline $\mathrm{V} 2$ & $1.380(3)$ & $0.0541(2)$ & $14.7(7)$ & 1536 & 1647 & 7.2 & 573 & 706 & 23.2 \\
\hline V3 & $0.979(2)$ & $0.0245(1)$ & $28(2)$ & 1911 & 2161 & 13.1 & 1072 & 1168 & 11.0 \\
\hline $\mathrm{V} 4$ & $1.245(3)$ & $0.045(2)$ & $23(1)$ & 1998 & 1956 & 2.1 & 866 & 961 & 11.0 \\
\hline $\mathrm{V} 5$ & $0.955(4)$ & $0.056(3)$ & $19(1)$ & 2348 & 2380 & 1.4 & 1005 & 1179 & 17.3 \\
\hline $\mathrm{V} 6^{*}$ & $1.195(7)$ & $0.103(5)$ & $34(2)$ & 2244 & 2220 & 1.1 & 1351 & 1407 & 4.1 \\
\hline $\mathrm{V} 7^{* *}$ & $1.08(1)$ & $0.129(7)$ & $27(1)$ & 3398 & 3314 & 2.5 & 2060 & 1930 & 6.3 \\
\hline
\end{tabular}



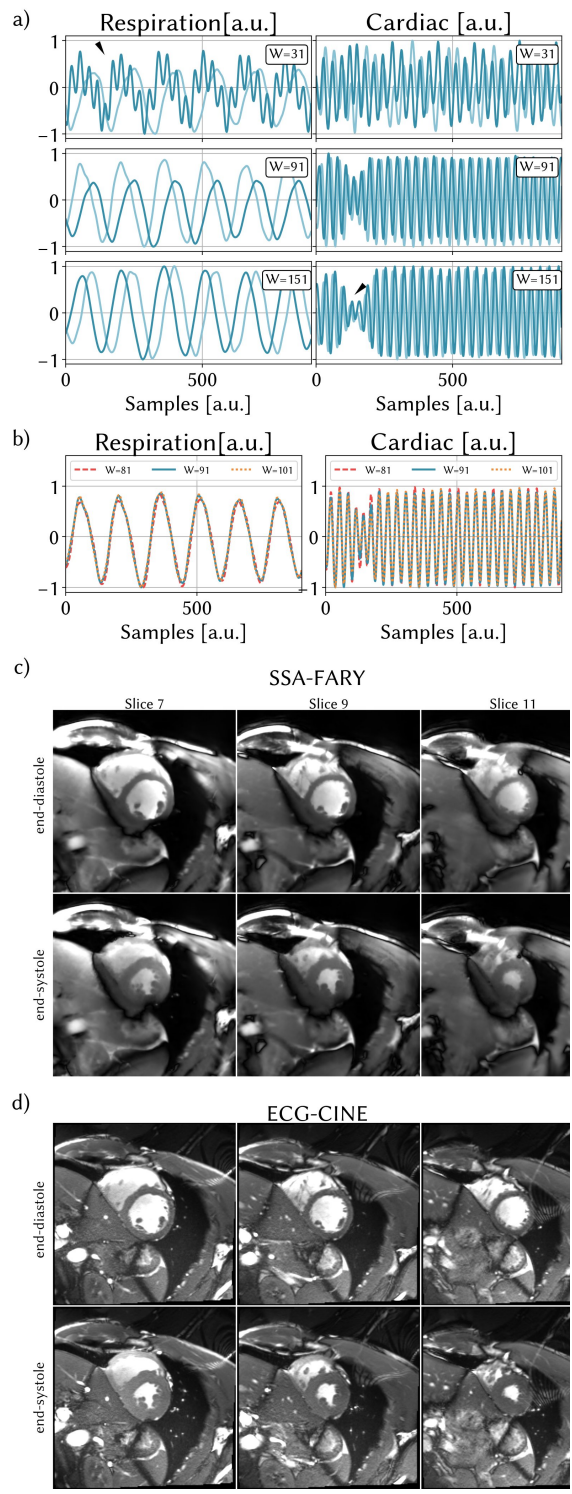

Figure 6: SSA-FARY self-gating signals for different window sizes from a SOS bSSFP acquisition and representative slices from a 3D PICS reconstructions for volunteer V5 in comparison to a conventional CINE scan. (a) A zoomed view on normalized EOF pairs representing respiratory and cardiac motion are depicted for window sizes $W=31, W=91$ and $W=151$. The black arrows indicate signal mixing (top-left) and signal loss (bottom right). (b) For window sizes $W=81, W=91$ and $W=101$, one EOF representing respiratory motion and one EOF representing cardiac motion are plotted in a zoomed view on top of each other. (c) shows three out of fourteen slices of an end-diastolic and endsystolic human heart after expiration. (d) depicts the corresponding vendor images for the ECG-triggered CINE breath-hold measurement. 


\section{Discussion}

We introduced a novel dimensionality reduction method dubbed "SSA-FARY", which is based on Singular Spectrum Analysis and showed that the proposed technique can successfully recover the cardiac and respiratory signal from the AC data of single-slice, SMS and SOS MRI measurements. Moreover, we have proposed an extended orthogonal projection to correct for system imperfections in the AC data.

AC correction The reasons for the oscillations in the AC data are many fold and according to our experience cannot be eliminated completely by techniques that correct for trajectory errors only [72, 73]. Particularly for bSSFP sequences, eddy current related dephasing of spins additionally compromises the data [74, 75], although this effect should be rather small for the tiny golden angle [61].

Zhang et al. [76 find this to be particularly problematic for 3D bSSFP imaging on a $3 \mathrm{~T}$ system and propose to eliminate these measurement errors by averaging over the central five samples of each spoke. However, we found this to produce even more oscillations in the $\mathrm{AC}$ data, which requires additional filtering. In contrast, the orthogonal projection used here can remove most of the signal perturbations directly. For constant increments of the projection angle, the correction can be thought of as a set of sharp band-stop or notch filters corresponding to higher-order harmonics of a base frequency, which can be calculated from the increment of the projection angle.

Strictly speaking, the AC correction is not mandatory for SSA-FARY as without filtering these spurious oscillations appear as additional EOFs. However, as these oscillations usually manifest distinctly in the AC region, the corresponding EOFs often possess the highest singular values. Consequently, they dominate the result of eq. (15) which reduces the significance and accuracy of the components of interest, i.e. cardiac and respiratory motion, and complicates the analysis. This can be easily avoided by using the proposed orthogonal projection, which corrects for first-order system imperfections.

Alternatively, advanced techniques to measure the trajectory error [77, 78, or higher-order system imperfection corrections could be utilized to account for the oscillations in the AC region [79, 80. Still, these approaches require additional hardware and/or sequence modifications, which limits their accessibility.

SSA-FARY The presented dimensionality reduction technique for time-series SSA-FARY can be considered a PCA applied to a time-delayed embedding of the AC data to exploit the locally low-rankness of dynamic time series. The Block-Hankel matrix $\boldsymbol{A}$ (eq. (8)) consists of shifted segments of the original

time series. Its covariance matrix $C_{t}^{t^{\prime}}=A_{t}\left(A^{H}\right)^{t^{\prime}}$ (eq. 117) is an array of scalar products specifying the correlation of all pairs of multi-channel segments in the embedding space. The considerable redundancy in the correlations of the segments in the presence of (quasi) repetitive oscillations, e.g. cardiac and respiratory motion, causes $C$ to have low-rank. By exploiting not only spatial 
but also these temporal correlations, SSA-FARY performs better in recognizing temporal patterns than classical PCA and allows the separation of trend, oscillations and noise from the signal. This was successfully demonstrated on numerical simulations of superposed and spoiled sinusoidal time series. In the actual in vivo measurements the respiratory and cardiac motion could be detected and clearly separated for all investigated sequence types.

To yield comparable results, methods like classical PCA must be combined with various pre- and post-processing techniques such as coil-selection or coilclustering [23], (iterative) band-pass filtering [50] and signal smoothing [20], which - especially for small AC regions - may be unstable and demand further manual tuning. By contrast, in SSA-FARY these steps are implicitly integrated and therefore surplus to requirement. Moreover, since SSA-FARY preserves phase information by producing quadrature pairs it allows for direct binning, which also renders the otherwise mandatory peak detection obsolete [10.

We demonstrated the high quality of the SSA-FARY gating signals by comparison with a real-time reconstruction, a respiratory belt and ECG triggers. Moreover, at free breathing and at a considerably lower acquisition time SSAFARY achieved a reconstruction quality that comes close to the results of the ECG-triggered CINE breath-hold scans. The analyzed left-ventricular bloodpool area of SSA-FARY reconstructions mostly corresponds well to the CINE results for end-diastole, whereas larger uncertainties were found for end-systole. Here, because the left-ventricular blood-pool area is significantly decreased compared to end-diastolic states, any deviation will result in larger relative errors. One reason might be that due to the high acceleration factor the short endsystolic phase is not perfectly resolved. In addition, some discrepancy between a breath-hold scan and a gated free-breathing scan is expected, since it cannot be guaranteed that the selected respiratory bins exactly matches the anatomical state of the breath-hold scan.

As the aim of this manuscript was the introduction of the self-gating technique, we did not fully optimize the sequence and reconstruction parameters, particularly the number of cardiac and respiratory bins, the regularization values of the ADMM and the undersampling scheme of the SOS sequence. The parameter tuning and the setup of a clinically applicable protocol is left for future investigations.

In single-slice imaging we only use a single sample per time step for autocalibration and despite the proportionally large window size, which reduces statistical significance, SSA-FARY yields reliable results. Still, SSA-FARY tends to be more resilient when multiple partitions are used and thus more AC data is available, as in SMS or SOS experiments, or when more samples relative to the window size are used for auto-calibration. For SMS or SOS imaging a bSSFP sequence is recommended since for RF-spoiled gradient-echo imaging a loss of contrast in systolic phases can occur due to pre-saturated blood flowing in from other slices, see Fig. 5. Note, however, that bSSFP sequences suffer from SAR limitations due to the increased flip-angle of the RF pulse and are prone to banding artifacts when no proper shimming is conducted.

Due to the zero-padding operation, eq. (7), and the subsequent Hankeliza- 
tion, eq. (8), the first and last samples in the SSA-FARY EOFs suffer from slight approximation errors. Still, for all presented experiments and analysis we did not discard these samples.

Occasionally, the trend was not completely separated from the EOFs for cardiac and respiratory motion, which we fixed by standardly using a moving average filter. The reason for the incomplete separation of trend can be understood by considering eq. 14, which relates the window size $W$ to the frequency bandwidth $\delta f_{\mathrm{B}}$ of the eigenfilters $\boldsymbol{V}$ generating the EOFs $\boldsymbol{U}$. Our default choice of window size and sampling rate corresponds to $\delta f_{\mathrm{B}} \approx 0.35 \mathrm{~Hz}$ for all measurements and sequences. If we choose $W$ too small, the EOFs capture a wider range of frequencies, which can result in a mixing of trend and oscillations. Similarly, if the respiration frequency (usually $f_{\text {resp }} \approx 0.3 \mathrm{~Hz}$ ) and cardiac frequency (usually $f_{\text {resp }} \approx 1.0 \mathrm{~Hz}$ ) happen to be spectrally close to one another, a very small window and the corresponding large frequency pass-band of the filters can hinder a proper separation, as it is the case in Fig. (6a), $W=31$ ). Then again, if we choose a very large $W$, the frequency response $f_{B}$ of the filters can be too narrow. Thus, possible frequency variations in the cardiac and respiratory motion can no longer be captured by a single (band-limited) EOF, which results in signal voids and/or the generation of additional EOFs representing higher order harmonics. Detecting these related EOFs corresponds to the 'Grouping' step of conventional SSA 81. In the cardiac signal of Fig. (6a) such a signal void caused by a cardiac frequency variation of $\approx 0.3 \mathrm{~Hz}$ can be observed for $W=151$, whereas the signal can still be adequately captured with $W=91$. As a summary, we found $\delta f_{\mathrm{B}} \approx 0.35 \mathrm{~Hz}$ to be a robust choice and we propose to choose the window size accordingly using eq. (14).

The computational bottle-neck of SSA-FARY is the SVD of $\boldsymbol{C}$ of size $\left[N_{t} \times\right.$ $N_{t}$. Especially for single-slice imaging, the window size $W$ required to obtain $\delta f_{\mathrm{B}} \approx 0.35 \mathrm{~Hz}$ and the corresponding number of AC samples $N_{t}$ to obtain good results is relatively large. Hence, the decomposition of $\boldsymbol{C}$ is rather timeconsuming. Nevertheless, there are approaches to significantly speed up the decomposition stage [82, 83].

SSA-FARY reliably detected the EOF pairs corresponding to cardiac and respiratory motion, which for $\mathrm{RF}$-spoiled gradient echo measurements usually possess the highest singular values. It is, however, not determined that the two largest components belong to the cardiac or the respiratory motion. For bSSFP sequences, which are generally more prone to system imperfections, we frequently found other components such as trends to have high singular values, too. Although for this study the components used for gating were chosen by visual inspection of the EOFs, it is fairly simple to automatize the assignment using a frequency analysis.

If one finds the separation of SSA-FARY to work insufficiently, a variation of the window size usually helps to recover a suitable gating result. Still, for patients with strong cardiac or respiratory frequency variations, highly nonperiodic respiratory motion or arrhythmia the results of the presented SSAFARY method might still be insufficient. In this case various promising extensions of SSA(-FARY) exist to improve the results, e.g. 'Nonlinear Laplacian 
Spectral Analysis', 'Sliding SSA', 'Oblique SSA', 'Nested SSA' and their combinations [84, 85, 47.

Outlook The property of SSA-FARY to not only reliably separate cardiac and respiratory motion but also to extract the trend in data, suggests further applications. In dynamic contrast-enhanced MRI, SSA-FARY could replace spline-fitting [20] for separating motion and contrast enhancement. Furthermore, preliminary results suggest that the $\mathrm{T} 1$ decay in inversion recovery sequences is detected as an individual component and separated from the motion signals, which would enable the simultaneous reconstruction of parameter maps and self-gated anatomical motion [86].

Last but not least we want to mention that the zero-padding approach used in SSA-FARY is not limited to self-gated MRI but turns multi-variate SSA into a general dimensionality reduction method, which might be useful for many other problems related to time-series analysis.

\section{Conclusion}

We have introduced a novel SSA-based dimensionality reduction method called SSA-FARY. Its intuitive approach, the easy implementation and its capability to separate cardiac and respiratory motion as well as trend makes it a promising approach for MRI self-gating, particularly when it is combined with efficient data acquisition schemes and a state-of-the-art image reconstruction technique.

\section{Acknowledgement}

The orthogonality projection to correct the AC data was originally conceived by Dr. Anthony G. Christodoulou (Biomedical Imaging Research Institute, Cedars-Sinai Medical Center). We extended it by including the higher-order harmonics.

Supported by the DZHK (German Centre for Cardiovascular Research), the Physics-to-Medicine Initiative Göttingen (LM der Niedersächsischen Vorab), the DFG (German Research Foundation) under grant UE 189/1-1, and the Studienstiftung des deutschen Volkes. We gratefully acknowledge the support of the NVIDIA Corporation with the donation of one NVIDIA TITAN Xp GPU for this research.

\section{A Details for Numerical Simulations}

Here we present the values for the variables used in the numerical simulation (Fig. 2). The total duration $T=800$ [a.u.] consists of 1000 discrete samples. The other parameters can be found in Table 2 
Table 2: Variables and values for the numerical simulation.

\begin{tabular}{cc}
\hline Variable & Value \\
\hline$N_{c}$ & 30 \\
$A$ & 3 \\
$\phi_{a}$ & 0 \\
$\omega_{a}$ & $\frac{2 \pi}{80}$ \\
$B$ & 1 \\
$\phi_{b}$ & 0.5 \\
$\omega_{b}$ & $\frac{2 \pi}{30}$ \\
$\sigma_{\text {noise }}$ & 2 \\
$C$ & 1 \\
$\phi_{c}$ & 0 \\
$\omega_{c}$ & $\frac{2 \pi}{10}$ \\
{$\left[t_{1}, t_{2}\right]$} & {$[220,300]$} \\
$D_{1}$ & 0.15 \\
$D_{2}$ & 1.5 \\
$\xi$ & $3.75 \cdot 10^{-3}$ \\
$W$ & 101 \\
$\Phi$ & 2 \\
\hline
\end{tabular}

\section{References}

[1] A. B. Kerr, J. M. Pauly, B. S. Hu, K. C. Li, C. J. Hardy, C. H. Meyer, A. Macovski, and D. G. Nishimura, "Real-time interactive mri on a conventional scanner," Magn. Reson. Med., vol. 38, no. 3, pp. 355-367, 1997.

[2] P. C. Yang, A. B. Kerr, A. C. Liu, D. H. Liang, C. Hardy, C. H. Meyer, A. Macovski, J. M. Pauly, and B. S. Hu, "New real-time interactive cardiac magnetic resonance imaging system complements echocardiography," $J$. Am. Coll. Cardiol., vol. 32, no. 7, pp. 2049-2056, 1998.

[3] M. Uecker, S. Zhang, and J. Frahm, "Nonlinear inverse reconstruction for real-time MRI of the human heart using undersampled radial FLASH," Magn. Reson. Med., vol. 63, no. 6, p. 1456-1462, 2010.

[4] M. Uecker, S. Zhang, D. Voit, A. Karaus, K.-D. Merboldt, and J. Frahm, "Real-time MRI at a resolution of 20 ms," NMR Biomed., vol. 23, no. 8, pp. 986-994, 2010.

[5] R. L. Ehman, M. McNamara, M. Pallack, H. Hricak, and C. Higgins, "Magnetic resonance imaging with respiratory gating: techniques and advantages," Am. J. Roentgenol., vol. 143, no. 6, pp. 1175-1182, 1984.

[6] Y. L. Liu, S. J. Riederer, P. J. Rossman, R. C. Grim, J. P. Debbins, and R. L. Ehman, "A monitoring, feedback, and triggering system for 
reproducible breath-hold $\mathrm{mr}$ imaging," Magn. Reson. Med., vol. 30, no. 4, pp. 507-511, 1993.

[7] L. Guo, "Novel techniques in projection-based motion tracking in cardiac magnetic resonance imaging," Ph.D. dissertation, Johns Hopkins University, 2017.

[8] C. B. Higgins and H. Hricak, Magnetic resonance imaging of the body. Elsevier, 1987.

[9] R. Rokey, R. E. Wendt, and D. L. Johnston, "Monitoring of acutely iii patients during nuclear magnetic resonance imaging: Use of a time-varying filter electrocardiographic gating device to reduce gradient artifacts," Magn. Reson. Med., vol. 6, no. 2, pp. 240-245, 1988.

[10] A. C. Larson, R. D. White, G. Laub, E. R. McVeigh, D. Li, and O. P. Simonetti, "Self-gated cardiac cine MRI," Magn. Reson. Med., vol. 51, no. 1, pp. 93-102, 2004.

[11] W. Kim, C. Mun, D. Kim, and Z. Cho, "Extraction of cardiac and respiratory motion cycles by use of projection data and its applications to $\mathrm{nmr}$ imaging," Magn. Reson. Med., vol. 13, no. 1, pp. 25-37, 1990.

[12] M. E. Crowe, A. C. Larson, Q. Zhang, J. Carr, R. D. White, D. Li, and O. P. Simonetti, "Automated rectilinear self-gated cardiac cine imaging," Magn. Reson. Med., vol. 52, no. 4, pp. 782-788, 2004.

[13] A. C. Larson, P. Kellman, A. Arai, G. A. Hirsch, E. McVeigh, D. Li, and O. P. Simonetti, "Preliminary investigation of respiratory self-gating for free-breathing segmented cine MRI," Magn. Reson. Med., vol. 53, no. 1, pp. 159-168, 2005.

[14] S. Uribe, V. Muthurangu, R. Boubertakh, T. Schaeffter, R. Razavi, D. L. G. Hill, and M. S. Hansen, "Whole-heart cine MRI using real-time respiratory self-gating," Magn. Reson. Med., vol. 57, no. 3, pp. 606-613, 2007.

[15] M. Buehrer, J. Curcic, P. Boesiger, and S. Kozerke, "Prospective self-gating for simultaneous compensation of cardiac and respiratory motion," Magn. Reson. Med., vol. 60, no. 3, pp. 683-690, 2008.

[16] J. Paul, E. Divkovic, S. Wundrak, P. Bernhardt, W. Rottbauer, H. Neumann, and V. Rasche, "High-resolution respiratory self-gated golden angle cardiac MRI: comparison of self-gating methods in combination with k-t SPARSE SENSE," Magn. Reson. Med., vol. 73, no. 1, pp. 292-298, 2015.

[17] J. Pang, B. Sharif, Z. Fan, X. Bi, R. Arsanjani, D. S. Berman, and D. Li, "Ecg and navigator-free four-dimensional whole-heart coronary mra for simultaneous visualization of cardiac anatomy and function," Magn. Reson. Med., vol. 72, no. 5, pp. 1208-1217, 2014. 
[18] M. Kirby, Geometric Data Analysis: An Empirical Approach to Dimensionality Reduction and the Study of Patterns. John Wiley \& Sons, Inc., 2000.

[19] Z. Deng, J. Pang, W. Yang, Y. Yue, B. Sharif, R. Tuli, D. Li, B. Fraass, and Z. Fan, "Four-dimensional mri using three-dimensional radial sampling with respiratory self-gating to characterize temporal phase-resolved respiratory motion in the abdomen," Magn. Reson. Med., vol. 75, no. 4, pp. 1574-1585, 2016.

[20] L. Feng, L. Axel, H. Chandarana, K. T. Block, D. K. Sodickson, and R. Otazo, "XD-GRASP: Golden-angle radial MRI with reconstruction of extra motion-state dimensions using compressed sensing," Magn. Reson. Med., vol. 75, no. 2, pp. 775-788, 2016.

[21] S. Rosenzweig, H. C. M. Holme, N. Scholand, R. N. Wilke, and M. Uecker, "Self-gated and real-time simultaneous multi-slice cardiac mri from the same acquisition," in Proc. Intl. Soc. Mag. Reson. Med., vol. 26, Paris, 2018, p. 0209.

[22] Y. Gao, Z. Zhou, F. Han, P. J. Finn, and P. Hu, "Improved respiratory motion self-gating in cardiovascular mri," J. Cardiov. Magn. Reson., vol. 18, no. 1, p. P7, 2016.

[23] T. Zhang, J. Y. Cheng, Y. Chen, D. G. Nishimura, J. M. Pauly, and S. S. Vasanawala, "Robust self-navigated body mri using dense coil arrays," Magn. Reson. Med., vol. 76, no. 1, pp. 197-205, 2016.

[24] K. Fukunaga, Introduction to statistical pattern recognition. Elsevier, 2013.

[25] N. Golyandina, V. Nekrutkin, and A. A. Zhigljavsky, Analysis of time series structure: SSA and related techniques. Chapman and Hall/CRC, 2001.

[26] F. Takens, "Detecting strange attractors in turbulence," in Dynamical Systems and Turbulence (Warwick 1980), vol. 898. Berlin, Heidelberg: Springer, Berlin, Heidelberg, 1981, pp. 366-381.

[27] D. S. Broomhead and G. P. King, "Extracting qualitative dynamics from experimental data," Physica D, vol. 20, no. 2-3, pp. 217-236, 1986.

[28] R. Vautard and M. Ghil, "Singular spectrum analysis in nonlinear dynamics, with applications to paleoclimatic time series," Physica D, vol. 35, no. 3, pp. 395-424, 1989.

[29] R. Vautard, P. Yiou, and M. Ghil, "Singular-spectrum analysis: A toolkit for short, noisy chaotic signals," Physica D, vol. 58, no. 1-4, pp. 95-126, 1992.

[30] D. S. Broomhead and G. P. King, "On the qualitative analysis of experimental dynamical systems," in Nonlinear Phenomena and Chaos, S. Sarkar, Ed. Bristol, England: Adam Hilger, 1986, pp. 113-144. 
[31] A. Hannachi, I. T. Jolliffe, and D. B. Stephenson, "Empirical orthogonal functions and related techniques in atmospheric science: A review," Int. J. Climatol., vol. 27, no. 9, pp. 1119-1152, 2007.

[32] K. Koelle, X. Rodó, M. Pascual, M. Yunus, and G. Mostafa, "Refractory periods and climate forcing in cholera dynamics," Nature, vol. 436, no. 7051, p. 696, 2005.

[33] I. A. Rezek and S. J. Roberts, "Stochastic complexity measures for physiological signal analysis," IEEE Trans. Biomed. Eng., vol. 45, no. 9, pp. 1186-1191, 1998.

[34] U. Kumar and V. Jain, "Time series models (grey-markov, grey model with rolling mechanism and singular spectrum analysis) to forecast energy consumption in india," Energy, vol. 35, no. 4, pp. 1709-1716, 2010.

[35] C. L. Wu, K. Chau, and Y. Li, "Methods to improve neural network performance in daily flows prediction," J. Hydrol., vol. 372, no. 1, pp. 80-93, 2009.

[36] H. Janssens, S. Hou, J. Jaeger, A.-R. Kim, E. Myasnikova, D. Sharp, and J. Reinitz, "Quantitative and predictive model of transcriptional control of the drosophila melanogaster even skipped gene," Nat. Genet., vol. 38, no. 10, p. 1159, 2006.

[37] L. Feng, L. Axel, L. A. Latson, J. Xu, D. K. Sodickson, and R. Otazo, "Compressed sensing with synchronized cardio-respiratory sparsity for free-breathing cine mri: initial comparative study on patients with arrhythmias," J. Cardiov. Magn. Reson., vol. 16, no. 1, p. O17, 2014.

[38] S. Rosenzweig, N. Scholand, H. Holme, and M. Uecker, "Robust cardiac and respiratory self-gating using an adapted singular spectrum analysis (ssa-fari): Application to simultaneous-multi-slice imaging," in Proc. Intl. Soc. Mag. Reson. Med., vol. 27, Montreal, 2019, p. 2444.

[39] S. Rosenzweig, N. Scholand, H. Holme, and M. Uecker, "Robust cardiac and respiratory self-gating using an adapted singular spectrum analysis," in Proc. Soc. Cardiovasc. Magn. Reson., vol. 22, Bellevue, 2019, p. 549871.

[40] N. A. Campbell and W. R. Atchley, "The geometry of canonical variate analysis," Syst. Biol., vol. 30, no. 3, pp. 268-280, 1981.

[41] M. Uecker, P. Lai, M. J. Murphy, P. Virtue, M. Elad, J. M. Pauly, S. S. Vasanawala, and M. Lustig, "ESPIRiT - an eigenvalue approach to autocalibrating parallel MRI: where SENSE meets GRAPPA," Magn. Reson. Med., vol. 71, no. 3, pp. 990-1001, 2014.

[42] T. Harris and H. Yuan, "Filtering and frequency interpretations of singular spectrum analysis," Physica D, vol. 239, no. 20-22, pp. 1958-1967, 2010. 
[43] K. Kume, "Interpretation of singular spectrum analysis as complete eigenfilter decomposition," Adv. Adapt. Data Anal., vol. 4, no. 04, p. 1250023, 2012.

[44] M. C. Leles, A. S. Cardoso, M. G. Moreira, H. N. Guimarães, C. M. Silva, and A. Pitsillides, "Frequency-domain characterization of singular spectrum analysis eigenvectors," in IEEE Intern. Symp. Signal Process. and Info. Tech. (ISSPIT). Limassol: IEEE, 2016, pp. 22-27.

[45] S. Xu, H. Hu, L. Ji, and P. Wang, "Embedding dimension selection for adaptive singular spectrum analysis of eeg signal," Sensors, vol. 18, no. 3, p. $697,2018$.

[46] G. Plaut and R. Vautard, "Spells of low-frequency oscillations and weather regimes in the northern hemisphere," J. Atmos. Sci., vol. 51, no. 2, pp. 210-236, 1994.

[47] N. Golyandina and A. Shlemov, "Variations of singular spectrum analysis for separability improvement: non-orthogonal decompositions of time series," Stat. Interface, vol. 8(3), pp. 277-294, 2015.

[48] A. Groth and M. Ghil, "Multivariate singular spectrum analysis and the road to phase synchronization," Phys. Rev. E, vol. 84, no. 3, p. 036206, 2011.

[49] M. Ghil, M. R. Allen, M. D. Dettinger, K. Ide, D. Kondrashov, M. E. Mann, A. W. Robertson, A. Saunders, Y. Tian, F. Varadi, and P. Yiou, "Advanced spectral methods for climatic time series," Rev. Geophys., vol. 40, no. 1, pp. 3-1-3-41, 2002.

[50] J. Liu, P. Spincemaille, N. C. F. Codella, T. D. Nguyen, M. R. Prince, and Y. Wang, "Respiratory and cardiac self-gated free-breathing cardiac CINE imaging with multiecho 3D hybrid radial SSFP acquisition," Magn. Reson. Med., vol. 63, no. 5, pp. 1230-1237, 2010.

[51] K. Nehrke, P. Bornert, D. Manke, and J. C. Bock, "Free-breathing cardiac mr imaging: study of implications of respiratory motion - initial results," Radiology, vol. 220, no. 3, pp. 810-815, 2001.

[52] I. Burger and E. M. Meintjes, "Elliptical subject-specific model of respiratory motion for cardiac mri," Magn. Reson. Med., vol. 70, no. 3, pp. 722-731, 2013.

[53] P. Dasari, K. Johnson, J. Dey, C. Lindsay, M. S. Shazeeb, J. M. Mukherjee, S. Zheng, and M. A. King, "Mri investigation of the linkage between respiratory motion of the heart and markers on patient's abdomen and chest: Implications for respiratory amplitude binning list-mode pet and spect studies," IEEE Trans. Nucl. Sci., vol. 61, no. 1, pp. 192-201, 2014. 
[54] P. K. Dasari, A. Könik, P. H. Pretorius, K. L. Johnson, W. P. Segars, M. S. Shazeeb, and M. A. King, "Correction of hysteretic respiratory motion in spect myocardial perfusion imaging: Simulation and patient studies," Med. Phys., vol. 44, no. 2, pp. 437-450, 2017.

[55] S. Rosenzweig, H. C. M. Holme, and M. Uecker, "Simple auto-calibrated gradient delay estimation from few spokes using radial intersections (ring)," Magn. Reson. Med., vol. 81, no. 3, pp. 1898-1906, 2019.

[56] S. Rosenzweig, H. Holme, and M. Uecker, "Simple auto-calibrated gradient delay estimation from few spokes using radial intersections (ring) for interactive real-time mri," in Proc. Intl. Soc. Mag. Reson. Med., vol. 27, Montreal, 2019, p. 445.

[57] V. Roeloffs, D. Voit, and J. Frahm, "Spoiling without additional gradients: Radial FLASH MRI with randomized radiofrequency phases," Magn. Reson. Med., vol. 75, no. 5, pp. 2094-2099, 2016.

[58] L. Feng, T. Zhao, H. Chandarana, D. K. Sodickson, and R. Otazo, "Motion-resolved golden-angle radial sparse mri using variable-density stack-of-stars sampling," in Proc. Intl. Soc. Mag. Reson. Med., Singapore, 2016, p. 1091.

[59] Z. Zhou, F. Han, L. Yan, D. J. Wang, and P. Hu, "Golden-ratio rotated stack-of-stars acquisition for improved volumetric mri," Magn. Reson. Med., vol. 78, no. 6, pp. 2290-2298, 2017.

[60] S. Rosenzweig, H. C. M. Holme, R. N. Wilke, D. Voit, J. Frahm, and M. Uecker, "Simultaneous multi-slice mri using cartesian and radial flash and regularized nonlinear inversion: Sms-nlinv," Magn. Reson. Med., vol. 79, no. 4, pp. 2057-2066, 2018.

[61] S. Wundrak, J. Paul, J. Ulrici, E. Hell, and V. Rasche, "A small surrogate for the golden angle in time-resolved radial mri based on generalized fibonacci sequences," IEEE Trans. Med. Imag., vol. 34, no. 6, pp. 12621269, 2015.

[62] B. Berman, Z. Li, M. Altbach, J. Galons, D. Martin, B. Dong, P. Sharma, B. Kalb, and A. Bilgin, "How to stack the stars: A variable center-dense k-space trajectory for 3d mri," in Proc. Intl. Soc. Mag. Reson. Med., Salt Lake City, 2013, p. 3829.

[63] M. Uecker, F. Ong, J. I. Tamir, D. Bahri, P. Virtue, J. Y. Cheng, T. Zhang, and M. Lustig, "Berkeley advanced reconstruction toolbox," in Proc. Intl. Soc. Mag. Reson. Med., vol. 23, Toronto, 2015, p. 2486.

[64] K. T. Block, M. Uecker, and J. Frahm, "Undersampled radial MRI with multiple coils. Iterative image reconstruction using a total variation constraint," Magn. Reson. Med., vol. 57, no. 6, p. 1086-1098, 2007. 
[65] S. Boyd, N. Parikh, E. Chu, B. Peleato, and J. Eckstein, "Distributed Optimization and Statistical Learning via the Alternating Direction Method of Multipliers," Found. Trends Mach. Learn., vol. 3, no. 1, pp. 1-122, 2011.

[66] J. Y. Cheng, T. Zhang, M. T. Alley, M. Uecker, M. Lustig, J. M. Pauly, and S. S. Vasanawala, "Comprehensive multi-dimensional mri for the simultaneous assessment of cardiopulmonary anatomy and physiology," Sci. Rep., vol. 7, no. 1, p. 5330, 2017.

[67] H. C. M. Holme, F. Ong, S. Rosenzweig, R. N. Wilke, M. Lustig, and M. Uecker, "ENLIVE: A Non-Linear Calibrationless Method for Parallel Imaging using a Low-Rank Constraint," in Proc. Intl. Soc. Mag. Reson. Med., vol. 25, Honolulu, 2017, p. 5160.

[68] F. Huang, S. Vijayakumar, Y. Li, S. Hertel, and G. R. Duensing, "A software channel compression technique for faster reconstruction with many channels," Magn. Reson. Imaging, vol. 26, no. 1, pp. 133-141, 2008.

[69] M. Buehrer, K. P. Pruessmann, P. Boesiger, and S. Kozerke, "Array compression for mri with large coil arrays," Magn. Reson. Med., vol. 57, no. 6, pp. 1131-1139, 2007.

[70] S. Rosenzweig, H. C. M. Holme, R. N. Wilke, and M. Uecker, "Simultaneous Multi-Slice Real-Time Imaging with Radial Multi-Band FLASH and Nonlinear Inverse Reconstruction," in Proc. Intl. Soc. Mag. Reson. Med., vol. 24, Honolulu, 2017, p. 0518.

[71] Y. Wang, S. J. Riederer, and R. L. Ehman, "Respiratory motion of the heart: kinematics and the implications for the spatial resolution in coronary imaging," Magn. Reson. Med., vol. 33, no. 5, pp. 713-719, 1995.

[72] N. Seiberlich, F. A. Breuer, M. Blaimer, K. Barkauskas, P. M. Jakob, and M. A. Griswold, "Non-cartesian data reconstruction using grappa operator gridding (grog)," Magn. Reson. Med., vol. 58, no. 6, pp. 1257-1265, 2007.

[73] A. Deshmane, M. Blaimer, F. Breuer, P. Jakob, J. Duerk, N. Seiberlich, and M. Griswold, "Self-calibrated trajectory estimation and signal correction method for robust radial imaging using grappa operator gridding," Magn. Reson. Med., vol. 75, no. 2, pp. 883-896, 2016.

[74] O. Bieri, M. Markl, and K. Scheffler, "Analysis and compensation of eddy currents in balanced ssfp," Magn. Reson. Med., vol. 54, no. 1, pp. 129-137, 2005.

[75] O. Bieri and K. Scheffler, "Flow compensation in balanced ssfp sequences," Magn. Reson. Med., vol. 54, no. 4, pp. 901-907, 2005.

[76] X. Zhang, G. Xie, N. Lu, Y. Zhu, Z. Wei, S. Su, C. Shi, F. Yan, X. Liu, B. Qiu, and Z. Fan, "3d self-gated cardiac cine imaging at 3 tesla using stack-of-stars bssfp with tiny golden angles and compressed sensing," Magn. Reson. Med., vol. 81, no. 5, pp. 3234-3244, 2019. 
[77] J. H. Duyn, Y. Yang, J. A. Frank, and J. W. van der Veen, "Simple correction method fork-space trajectory deviations in mri," J. Magn. Reson., vol. 132, no. 1, pp. 150 - 153, 1998.

[78] C. Barmet, N. D. Zanche, and K. P. Pruessmann, "Spatiotemporal magnetic field monitoring for mr," Magn. Reson. Med., vol. 60, no. 1, pp. 187-197, 2008.

[79] S. J. Vannesjo, M. Haeberlin, L. Kasper, M. Pavan, B. J. Wilm, C. Barmet, and K. P. Pruessmann, "Gradient system characterization by impulse response measurements with a dynamic field camera," Magn. Reson. Med., vol. 69, no. 2, pp. 583-593, 2013.

[80] M. Stich, T. Wech, A. Slawig, R. Ringler, A. Dewdney, A. Greiser, G. Ruyters, T. A. Bley, and H. Köstler, "Gradient waveform pre-emphasis based on the gradient system transfer function," Magn. Reson. Med., 2018.

[81] N. Golyandina and A. Zhigljavsky, Singular Spectrum Analysis for time series. Springer Science \& Business Media, 2013.

[82] A. Korobeynikov, "Computation- and space-efficient implementation of ssa," Stat. Interface, vol. 3, no. 3, pp. 357-368, 2010.

[83] M. Leles, J. Sansão, L. Mozelli, and H. Guimarães, "A new algorithm in singular spectrum analysis framework: The overlap-ssa (ov-ssa)," SoftwareX, vol. 8, pp. 26-32, 2018.

[84] D. Giannakis and A. J. Majda, "Nonlinear laplacian spectral analysis for time series with intermittency and low-frequency variability," PNAS, vol. 109, no. 7, pp. 2222-2227, 2012.

[85] J. Harmouche, D. Fourer, F. Auger, P. Borgnat, and P. Flandrin, "The sliding singular spectrum analysis: a data-driven nonstationary signal decomposition tool," IEEE Trans. Signal Process., vol. 66, no. 1, pp. 251-263, 2018.

[86] A. G. Christodoulou, J. L. Shaw, C. Nguyen, Q. Yang, Y. Xie, N. Wang, and D. Li, "Magnetic resonance multitasking for motion-resolved quantitative cardiovascular imaging," Nat. Biomed. Eng., vol. 2, no. 4, p. 215, 2018. 


\title{
Supplementary Document - Cardiac and Respiratory Self-Gating in Radial MRI using an Adapted Singular Spectrum Analysis (SSA-FARY)
}

\author{
Sebastian Rosenzweig*1,2, Nick Scholand ${ }^{1,2}$, H. Christian M. \\ Holme $^{1,2}$, and Martin Uecker ${ }^{1,2}$ \\ ${ }^{1}$ Institute for Diagnostic and Interventional Radiology, University \\ Medical Center Göttingen, Göttingen, Germany \\ ${ }^{2}$ German Centre for Cardiovascular Research (DZHK), Partner \\ Site Göttingen, Göttingen, Germany
}

March 9, 2020

\begin{abstract}
This supplementary material provides additional information and experiments regarding the numerical simulation and the in vivo experiments.
\end{abstract}

\section{Numerical Simulations with Varying Simula- tion Parameters}

We demonstrate the effects of different parameter values on the numerical simulation. For each simulation, we keep the basic parameters as given in Table II of the main part of the manuscript and list all modified parameters in Table 1 of this supplementary material.

For Fig. (1), we moderately increase the noise variance and the amplitude of the spell and the trend. Consequently, the PCA performs even worse in detecting the individual oscillations, particularly for the oscillation with the higher frequency. In contrast, SSA-FARY still provides satisfying results.

\footnotetext{
*Sebastian Rosenzweig, University Medical Center Göttingen, Institute for Diagnostic and Interventional Radiology, Robert-Koch-Str. 40, 37075 Göttingen, Germany. sebastian.rosenzweig@med.uni-goettingen.de
} 
Table 1: Variables and values for the numerical simulation.

\begin{tabular}{ccc}
\hline & Variable & Value \\
\hline Fig. (1) & $\sigma_{\text {noise }}$ & 4 \\
& $C$ & 2 \\
& $D_{1}$ & 0.25 \\
& $D_{2}$ & 2.5 \\
Fig. (2) & $\sigma_{\text {noise }}$ & 7 \\
& $C$ & 3 \\
& $D_{1}$ & 0.35 \\
& $D_{2}$ & 3.5 \\
Fig. (3) & $\Phi$ & 3 \\
\hline
\end{tabular}

For Fig. (2), we further increase the noise variance and the amplitude of the spell and the trend. Here, the SSA-FARY result for the lower frequency oscillations are still acceptable, whereas the EOFs for the higher frequency oscillation are now also clearly corrupted.

For Fig. (3), the same parameters for noise variance, trend and spell are used as in the main part of the manuscript, but a higher frequency variation is simulated. The PCA result is comparable to the one in the main part of the manuscript, while the SSA-FARY results look still satisfying, yet slightly worse due to the limited frequency bandwidth of the EOFs, which is determined by the window size $W$. 

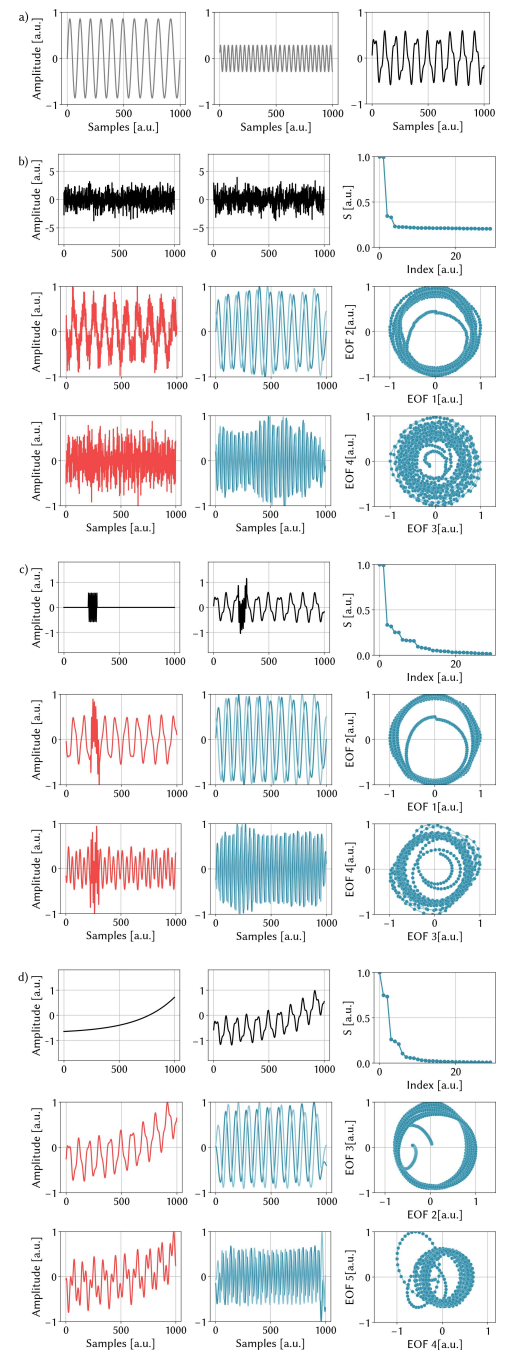

Figure 1: Comparison of PCA eigenvectors and SSA-FARY EOFs. In this example there is a moderate increase of the noise variance and the spell and trend amplitude compared to the main part of the manuscript. a) Frequency-modulated sinusoidal oscillations $(a(t)$ left, $b(t)$ center) used for the numerical simulations and weighted composite signal (right). b), c), d) Black: Different additional signals (left) - b) noise, c) oscillatory spell, d) exponential trend - added to the composite signal of a) and a selected channel of the resulting signal $X_{i}(t)$ (right). Red: First two eigenvectors of the PCA. Blue: SSA-FARY singular values $S$ (scree plot) and EOF pairs (amplitude-time plot and amplitude-amplitude scatter plot). All plots are normalized. 

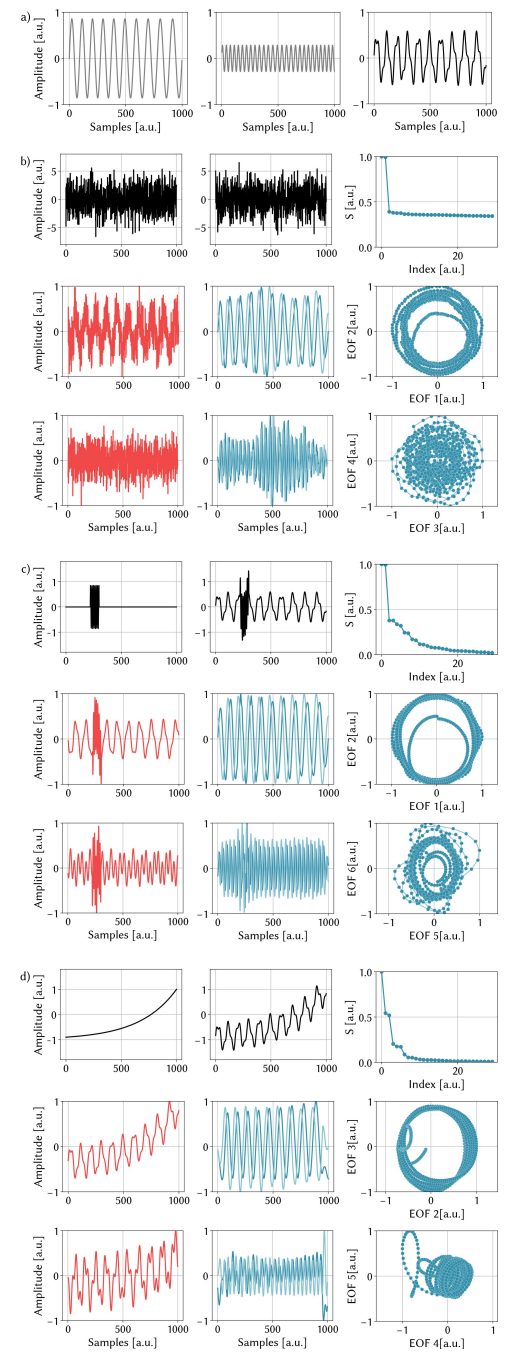

Figure 2: Comparison of PCA eigenvectors and SSA-FARY EOFs. In this example there is a strong increase of the noise variance and the spell and trend amplitude compared to the main part of the manuscript. See also caption of Fig. (1). 

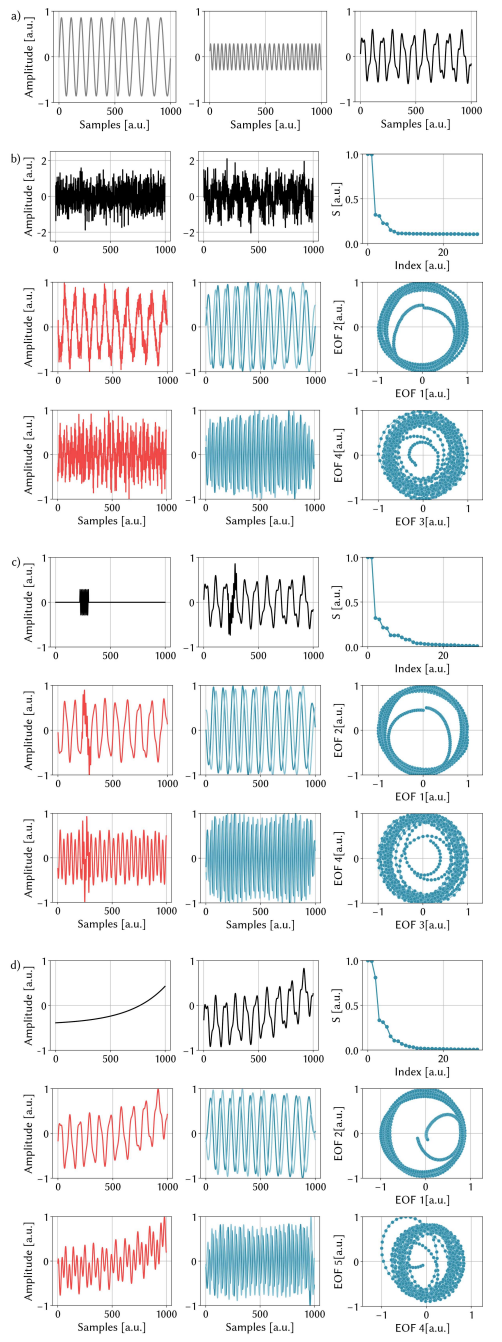

Figure 3: Comparison of PCA eigenvectors and SSA-FARY EOFs. In this example there is a moderate increase of the frequency variation compared to the main part of the manuscript. See also caption of Fig. (1). 


\section{SSA-FARY Gated bSSFP reconstruction us- ing conventional gridding}

While we have used only 30 seconds of data in the single-slice experiment described in the main part of the manuscript, we now apply SSA-FARY self-gating and PCA self-gating to the full 72 seconds of data. We use the same SSA-FARY window size $W=751$ and bin the data into 25 cardiac and 3 respiratory bins. On the SSA-FARY-gated data we perform a conventional gridding reconstruction followed by a root-sum-of-square coil combination.

Similar to the results of the main part of the manuscript, SSA-FARY outperforms the PCA self-gating, which fails to separate the cardiac from respiratory motion, see Fig. 4.

Because of the longer acquisition time and the reduced number of respiratory bins, each cardiac state contains sufficient spokes such that a conventional gridding reconstruction can be performed. While mild undersampling artifacts can be observed, the temporal dynamics are well resolved, see Fig. 4 and movie Mov5.

Note, that due to the limited number of respiratory bins we can't depict the matching respiratory state of the ECG-gated CINE breath-hold scan for the gridding reconstruction. 

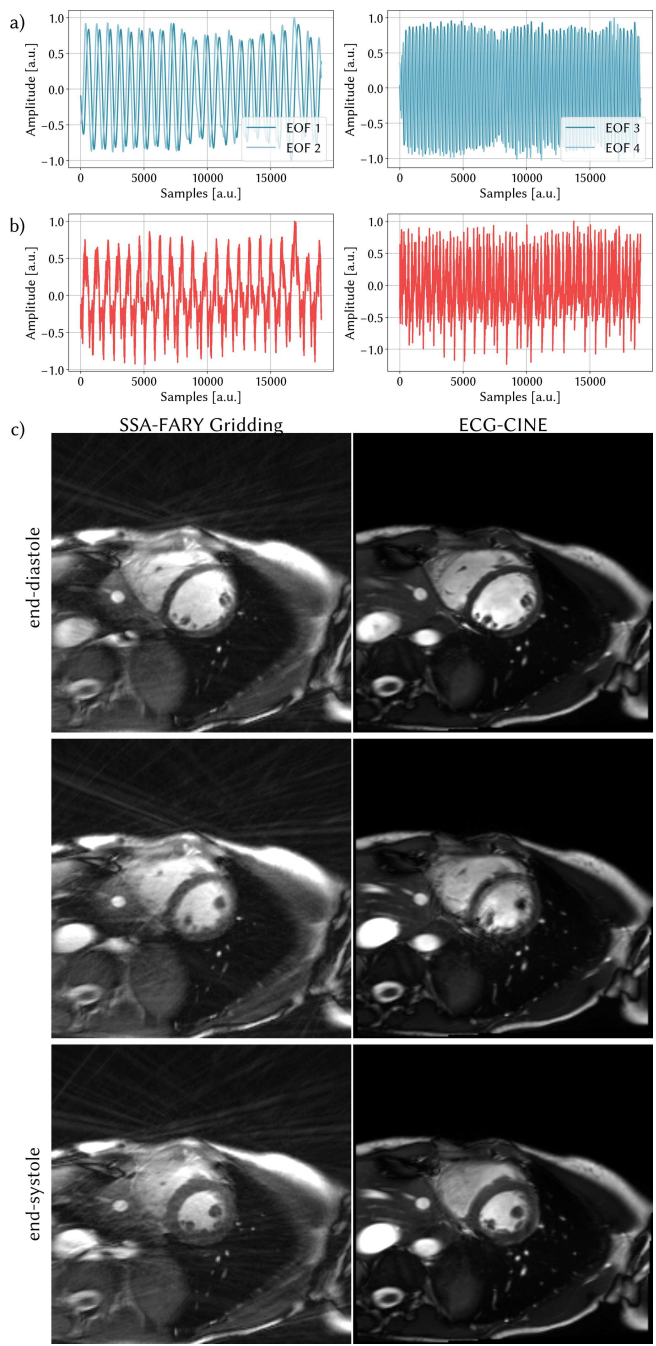

Figure 4: Self-gating signals and gridding reconstruction of a single-slice radial bSSFP aquisition in comparison to conventional CINE images. In (a) the two quadrature EOFs of SSA-FARY are plotted against time for the respiratory (left) and cardiac (right) motion, respectively. In (b) the first two eigenfunctions of the PCA are depicted. For (c) the self-gating signal of (a) was utilized. It shows three representative frames of the SSA-FARY-gated gridding reconstruction corresponding to three cardiac phases from end-systole to end-diastole for a single respiration state. As in the Figure 4 of the main manuscript, we also show vendor images of the ECG-triggered CINE breath-hold scan. 


\section{SSA-FARY Gated Reconstructions for a Single- Slice RF-Spoiled Gradient-Echo Sequence}

Similar to the bSSFP single-slice experiment of the main part of the manuscript, we perform a 30 second RF-spoiled gradient-echo scan $(\mathrm{TE} / \mathrm{TR}=1.63 / 2.60 \mathrm{~ms}$, flip angle $12^{\circ}$ ) with slice-thickness $7 \mathrm{~mm}$ of the human heart in short-axis view.

We compare the principal motion signals using PCA and SSA-FARY with window size $W=1171$, which covers a period of about $3 \mathrm{~s}$.

For each coil, Fig. 5 depicts the DC component of 100 consecutive spokes before and after the data correction using the orthogonal projection. Just as in Fig. 3 of the main part of the manuscript, some coils exhibit pronounced oscillations with approximately 15 samples per period, which is related to the seventh tiny golden angle. With the proposed correction method these oscillations can be eliminated.

Fig. 6 shows the self-gating signals generated with PCA and SSA-FARY. In SSA-FARY, the first two EOFs represent respiratory motion and the third and fourth EOF cardiac motion. The EOFs of the pairs are in quadrature and the motion signals are well separated. PCA fails in separating the cardiac from the respiratory motion and both principle components are spoiled by noise.

Fig. 6c shows six representative images of the SSA-FARY-gated reconstruction, three of which in end-systole and three in end-diastole. Depicted are three respiratory states from end-inspiration to end-expiration.

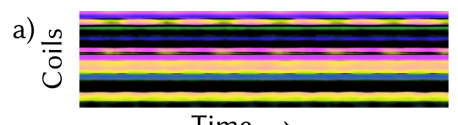

Time $\rightarrow$ b) $\frac{n}{3}$

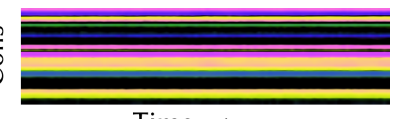

Figure 5: Snippet of the amplitude plot with color-coded phase of the DC samples used for auto-calibration of the single-slice reconstruction before (a) and after (b) the data correction using the orthogonal projection. The period length of the oscillations in (a) corresponds to 15 samples. 

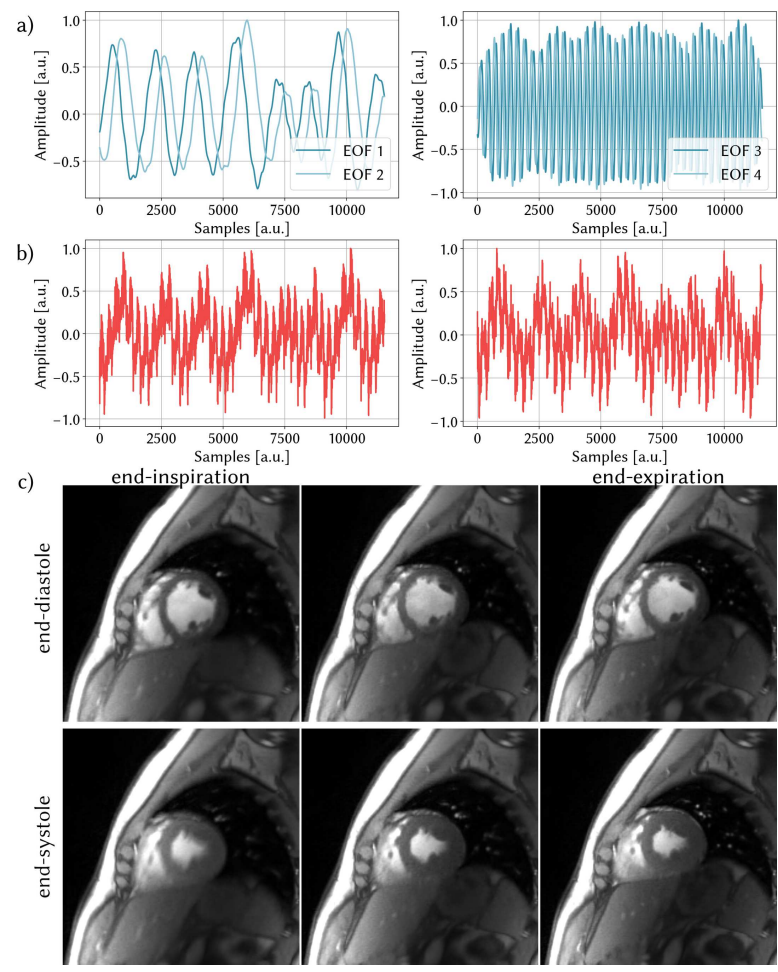

Figure 6: Self-gating signals and PICS reconstruction from a single-slice radial gradient-echo acquisition. In (a) the two quadrature EOFs of SSA-FARY are plotted against time for the respiratory (left) and cardiac (right) motion, respectively. In (b) the first two eigenfunctions of the PCA are depicted. In both, a superposition of the cardiac and respiratory motion can be perceived. For (c) the self-gating signal of (a) was utilized. It shows six representative frames corresponding to end-systole and end-diastole for end-inspiration, an intermittent state and end-expiration. 


\section{Respiratory Signal Comparison for Irregular Breathing}

We perform the same SMS measurement as described in the main part of the manuscript on another volunteer who happened to have an irregular breathing pattern during the measurement. We depict the results of the experiment in Fig. 7.

For all three slices, Fig. 7a shows 5 out of 25 cardiac phases in end-expiration, where the individual cardiac phases can be distinguished well. From the SMSNLINV real-time reconstruction of the full time series, we determine the actual motion of the lung by extracting a line from slice one which cuts the diaphragm in vertical direction, and use it as background for Fig. 7b. On top, we plot a respiratory EOF obtained using SSA-FARY. It shows good agreement with the diaphragm motion in the background.

We also depict the signal from the respiratory belt, which tends to be temporarily misaligned with the diaphragm position after deep inhalation.

For the purpose of clarity, we do not plot the quadrature pair belonging to the depicted EOF. Similar to Fig. 5 of the main part of the manuscript, the motion signals were scaled to match the amplitude of the diaphragm position displayed in the background image.

Deviations of the respiratory belt signal from the actual diaphragm position are not surprising. The correlation between external physiological motion and internal organ movement is subject dependent and patient specific signal processing can be required to account for the associated dephasing [1,2], which is specifically important for irregular breathing patterns [3, 4]. Moreover, it is a know issue that the signal from a respiratory belt may be imperfect [5]. Furthermore, it was shown that an internal navigator based on the diaphragmatic motion outperforms the respiratory belt [6]. Consequently, the respiratory belt is not widely used for respiratory gating in MRI [7].

This experiment shows that - even for irregular breathing - the respiratory gating signal obtained with SSA-FARY resembles the diaphragmatic position largely well both in phase and in amplitude and therefore constitutes a suitable surrogate for respiratory gating. 


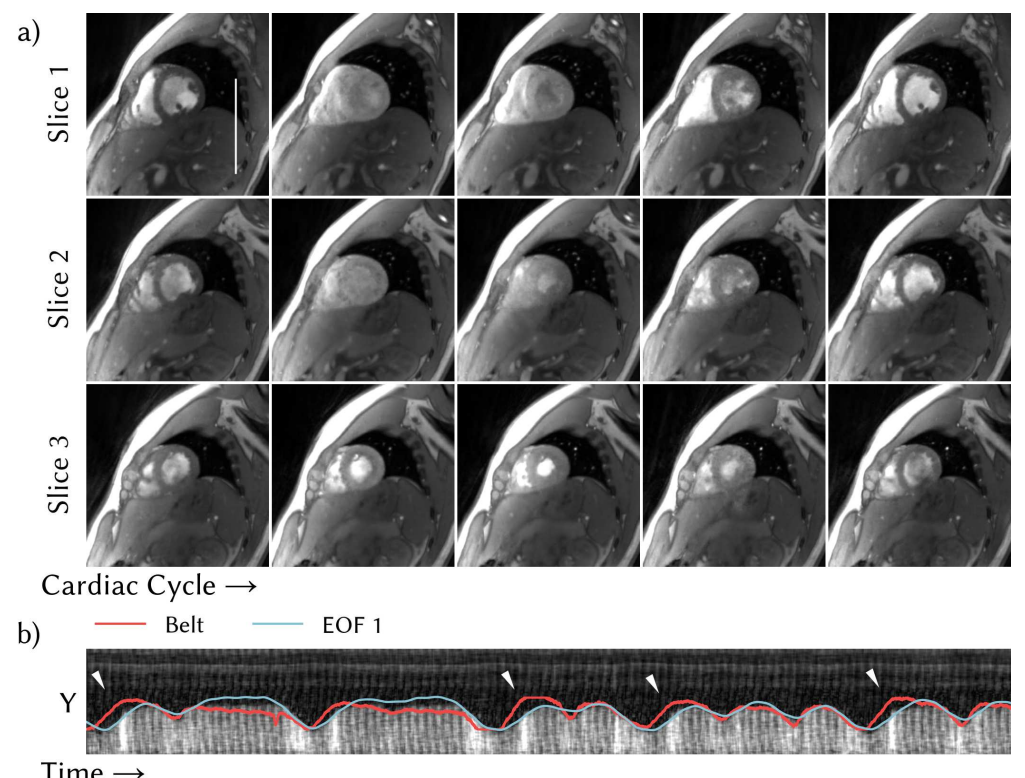

Figure 7: PICS reconstructions and self-gating signal of a radial SMS gradientecho acquisition. For all three slices, (a) shows 5 out of 25 cardiac phases in end-expiration. The background of (b) is extracted from a SMS-NLINV realtime reconstruction of the entire time series. It shows the temporal evolution of a vertical line (highlighted in white), placed on the diaphragm in slice one. As an overlay, the signal obtained from the respiratory belt and the EOF which is in phase with the lung motion is plotted. The signal from the respiratory belt shows some disparities compared to the real-time reconstruction after deep inhalation. This is indicated by the white arrows. 


\section{SSA-FARY Gated Reconstructions for Highly Irregular Breathing, Yawning and a Pronounced Heart Rate Variability.}

Here, we present more details on the gating and reconstruction of the Stack-ofStars measurements of volunteers V6 and V7.

Irregular breathing pattern Volunteer V6 shows a non-dictated highly irregular breathing pattern with periods of normal breathing, interrupted by various breath-hold intervals of up to approximately $12 \mathrm{~s}$. The default EOF bandwidth $\delta f_{\mathrm{B}} \approx 0.35 \mathrm{~Hz}$, which here corresponds to a window size of $W=91$, is too narrow to capture this complex breathing motion in a single EOF pair. In fact, SSA-FARY creates at least 6 EOFs to account for the respiratory motion. Consequently, the cardiac motion is only given by EOF 8 and EOF 9, see Fig. (8a). Since for respiratory binning a single EOF pair is required, we run SSAFARY again with reduced window size $W=51$ to increase the spectral width of the EOFs. This choice and the corresponding EOFs 2 and 3 allow for a proper binning of the respiratory motion. The window size of the moving average was adjusted to $L_{\mathrm{avg}}=600$ samples. The results for end-expiration are depicted in Fig. (8b), together with the corresponding slices of the ECG-CINE breath-hold measurement (8c).

Yawning and pronounced cardiac frequency variations Volunteer V7 admitted that he unintentionally yawned during the three minute measurement. The volunteer furthermore exhibits a highly irregular heartbeat with the heart rate standard deviation of $\bar{\sigma}_{\text {heart }}=0.129 \mathrm{~Hz}$ and heart rate jumps of up to $0.3 \mathrm{~Hz}$.

Using the window size $W=91$, the three yawns are very well reflected in the first EOF, while the respiration is represented by EOFs 2 and 3, see Fig. (9a). The first EOF was used to discard the intervals in which the volunteer yawned before binning, which reduces the for the reconstruction effectively available data about $30 \mathrm{~s}$. Note that the analysis for $\sigma_{\text {trig }}$ of Table I in the main part of the manuscript was performed using the full data-set.

Due to the high heart rate variability, we run SSA-FARY again with window size $W=21$ to increase the spectral width of the EOFs for cardiac binning. Although such a low window-size can lead to a mixing of respiratory and cardiac oscillations, as was shown in Fig. (6a) of the main part of the manuscript, this is not the case here and the determined gating signal is in good agreement with the ECG signal, see Table I of the main part of the manuscript. To better compensate the signal offset in the cardiac EOFs, which particularly arouse during the yawing periods, we adapted the moving average filter for the cardiac EOFs to $L_{\text {avg }}=30$. 
a)

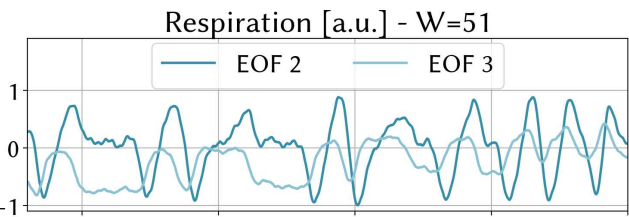

Cardiac [a.u.] - W=91

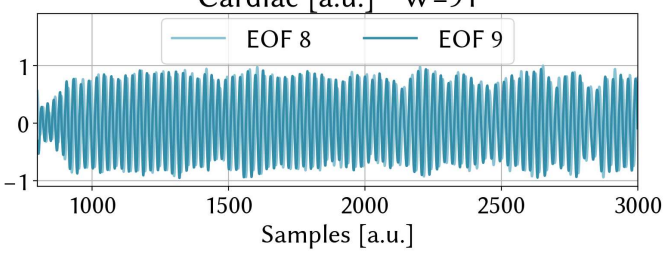

b)

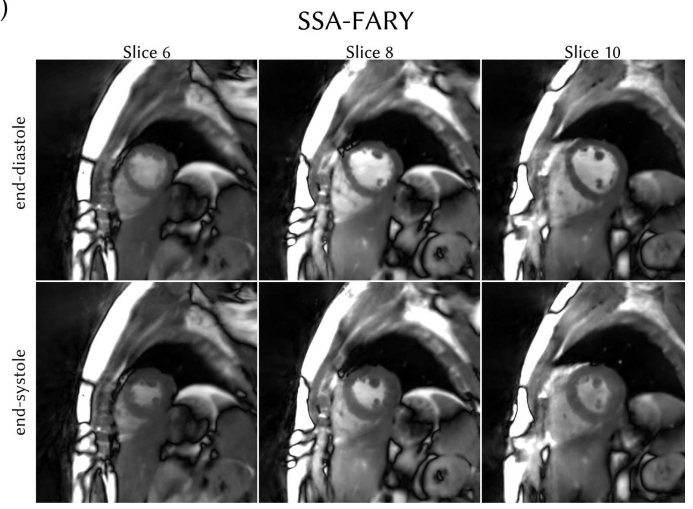

c)

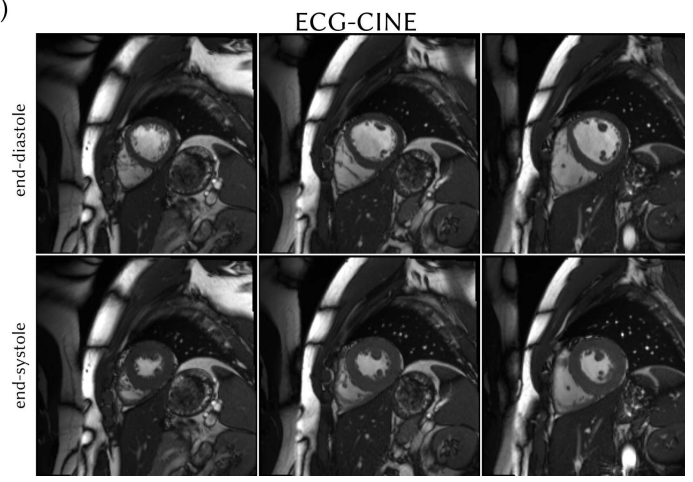

Figure 8: SSA-FARY self-gating signals and representative slices from a 3D PICS reconstruction of a SOS bSSFP acquisition for volunteer V6 in comparison to conventional CINE images. (a) depicts a zoomed view on normalized EOF pairs representing respiratory and cardiac motion using the window sizes $W=51$ and $W=91$, respectively. (b) shows three out of fourteen slices in end-diastole and end-systole after expiration. (c) shows the corresponding reconstructions for the ECG-triggered CINE breath-hold measurement. 
a)
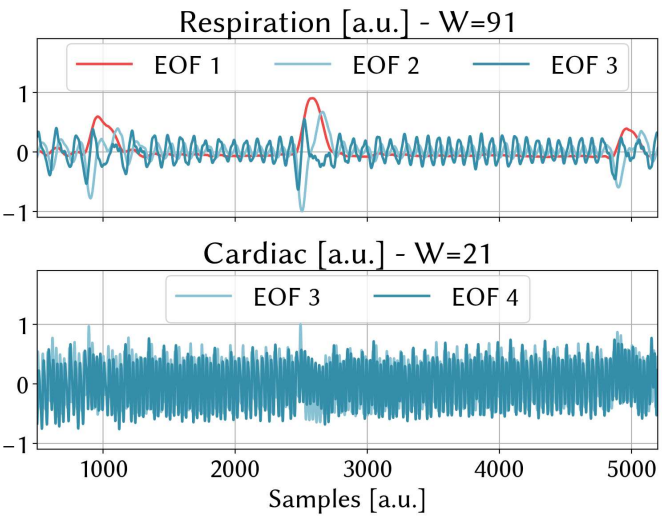

b)

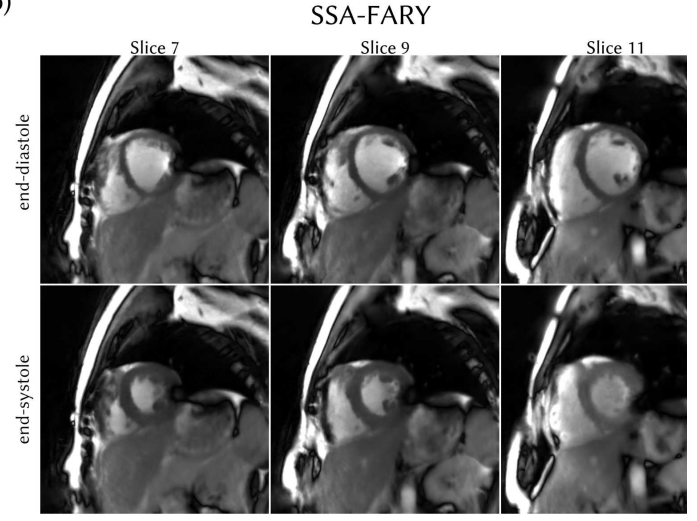

c)

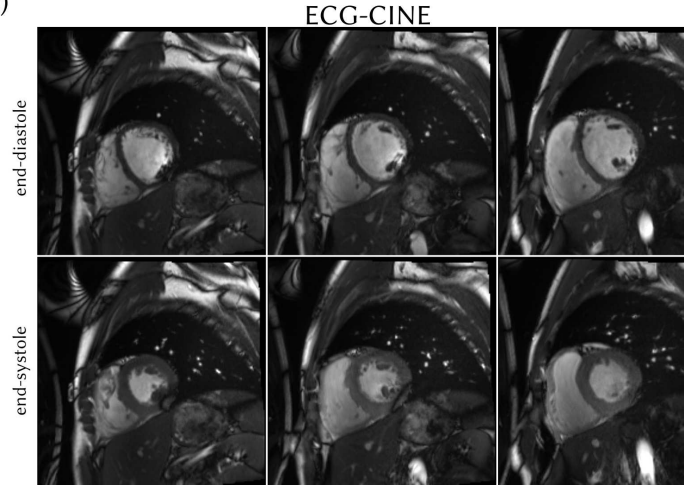

Figure 9: SSA-FARY self-gating signals and representative slices from a 3D PICS reconstruction of a SOS bSSFP acquisition for volunteer V7 in comparison to conventional CINE images. (a) A zoomed view on the normalized EOFs 1, 2 and 3 determined with window size $W=91$. While EOF 1 captures the yawning, the EOFs 2 and 3 represent breathing motion. For window size $W=21$, the EOFs 3 and 4 capture the cardiac motion. (b) shows three out of fourteen slices in end-diastole and end-systole after expiration. (c) shows the corresponding reconstructions for the ECG-triggered CINE breath-hold measurement. 


\section{Movies of the In Vivo Experiments}

For the single-slice bSSFP (Mov1-2) and RF-spoiled gradient-echo measurements (Mov3-4), we have attached two movie files, respectively. Mov1 and Mov3 show the cardiac cycle for end-expiration (left) and end-inspiration. Mov2 and Mov4 show the respiratory cycle for end-systole (left) and end-diastole. To account for the low number of respiratory bins, we have interpolated the frames in the respiratory resolved videos.

Mov5 shows a cardiac cycle of the gridding reconstruction.

Mov6 shows a cardiac cycle for all three slices of the SMS reconstruction from the main part of the manuscript at end-expiration (top) and end-inspiration.

Mov7 shows a cardiac cycle for all three slices of the SMS reconstruction with erratic breathing at end-expiration.

For all analyzed volunteers V1-V7 of the Stack-of-Stars measurements, we have attached a representative movie (Mov8 - Mov14) showing a cardiac cycle for 6 out of 14 slices in end-expiration. 


\section{References}

[1] A. T. Hess, E. M. Tunnicliffe, C. T. Rodgers, and M. D. Robson, "Diaphragm position can be accurately estimated from the scattering of a parallel transmit rf coil at 7 t," Magn. Reson. Med., vol. 79, no. 4, pp. 2164-2169, 2018.

[2] F. Odille, P.-A. Vuissoz, P.-Y. Marie, and J. Felblinger, "Generalized reconstruction by inversion of coupled systems (grics) applied to free-breathing mri," Magn. Reson. Med., vol. 60, no. 1, pp. 146-157, 2008.

[3] M. Henningsson, T. Hussain, M. S. Vieira, G. F. Greil, J. Smink, G. v. Ensbergen, G. Beck, and R. M. Botnar, "Whole-heart coronary mr angiography using image-based navigation for the detection of coronary anomalies in adult patients with congenital heart disease," J. Magn. Reson. Imaging, vol. 43, no. 4, pp. 947-955, 2016.

[4] C. Santelli, R. Nezafat, B. Goddu, W. J. Manning, J. Smink, S. Kozerke, and D. C. Peters, "Respiratory bellows revisited for motion compensation: preliminary experience for cardiovascular mr," Magn. Reson. Med., vol. 65, no. 4, pp. 1097-1102, 2011.

[5] M. Filipovic, P.-A. Vuissoz, A. Codreanu, M. Claudon, and J. Felblinger, "Motion compensated generalized reconstruction for free-breathing dynamic contrast-enhanced mri," Magn. Reson. Med., vol. 65, no. 3, pp. 812-822, 2011.

[6] G. Li, J. Wei, D. Olek, M. Kadbi, N. Tyagi, K. Zakian, J. Mechalakos, J. O. Deasy, and M. Hunt, "Direct comparison of respiration-correlated four-dimensional magnetic resonance imaging reconstructed using concurrent internal navigator and external bellows," Int. J. Radiat. Oncol. Biol. Phys., vol. 97, no. 3, pp. 596-605, 2017.

[7] J. R. McClelland, D. J. Hawkes, T. Schaeffter, and A. P. King, "Respiratory motion models: a review," Med. Image Anal., vol. 17, no. 1, pp. 19-42, 2013. 